\title{
The substellar mass function in $\sigma$ Orionis
}

\section{Optical, near-infrared and IRAC/Spitzer photometry of young cluster brown dwarfs and planetary-mass objects $\star, \star \star$}

\author{
J. A. Caballero ${ }^{1,2}$, V. J. S. Béjar ${ }^{3}$, R. Rebolo ${ }^{1,4}$, J. Eislöffel ${ }^{5}$, M. R. Zapatero Osorio ${ }^{1,6}$, R. Mundt $^{2}$, \\ D. Barrado y Navascués ${ }^{6}$, G. Bihain ${ }^{1,4}$, C. A. L. Bailer-Jones ${ }^{2}$, T. Forveille ${ }^{7,8}$, and E. L. Martín ${ }^{1,9}$ \\ 1 Instituto de Astrofísica de Canarias, Avenida Vía Láctea, 38200 La Laguna, Tenerife, Islas Canarias, Spain \\ 2 Max-Planck-Institut für Astronomie, Königstuhl 17, 69117 Heidelberg, Germany \\ e-mail: caballero@mpia.de \\ Proyecto Gran Telescopio Canarias, Instituto de Astrofísica de Canarias \\ 4 Consejo Superior de Investigaciones Científicas, Spain \\ Thüringer Landessternwarte, Sternwarte 5, 07778 Tautenburg, Germany \\ ${ }^{6}$ LAEFF-INTA, P.O. Box 50727, 28080, Madrid, Spain \\ 7 Canada-France-Hawaii Telescope Corporation, 65-1238 Mamalahoa Highway, Kamuela, HI96743, Hawai'i, USA \\ ${ }^{8}$ Laboratoire d'Astrophysique, Observatoire de Grenoble, BP 53, 38041 Grenoble Cedex 9, France \\ 9 University of Central Florida, Dept. of Physics, PO Box 162385, Orlando, FL 32816-2385, USA
}

Received 21 December 2006 / Accepted 12 April 2007

ABSTRACT

\begin{abstract}
Aims. We investigate the mass function in the substellar domain down to a few Jupiter masses in the young $\sigma$ Orionis open cluster $\left(3 \pm 2 \mathrm{Ma}, d=360_{-60}^{+70} \mathrm{pc}\right)$.

Methods. We have performed a deep $I J$-band search, covering an area of $790 \mathrm{arcmin}^{2}$ close to the cluster centre. This survey was complemented with an infrared follow-up in the $H K_{\mathrm{s}^{-}}$and Spitzer 3.6-8.0 $\mu \mathrm{m}$-bands. Using colour-magnitude diagrams, we have selected 49 candidate cluster members in the magnitude interval $16.1 \mathrm{mag}<I<23.0 \mathrm{mag}$.

Results. Accounting for flux excesses at $8.0 \mu \mathrm{m}$ and previously known spectral features of youth, we identify 30 objects as bona fide cluster members. Four are first identified from our optical-near infrared data. Eleven have most probable masses below the deuterium burning limit which we therefore classify as candidate planetary-mass objects. The slope of the substellar mass spectrum $\left(\Delta N / \Delta M \approx a M^{-\alpha}\right)$ in the mass interval $0.11 M_{\odot}<M<0.006 M_{\odot}$ is $\alpha=+0.6 \pm 0.2$. Any mass limit to formation via opacity-limited fragmentation must lie below $0.006 M_{\odot}$. The frequency of $\sigma$ Orionis brown dwarfs with circumsubstellar discs is $47 \pm 9 \%$.

Conclusions. The continuity in the mass function and in the frequency of discs suggests that very low-mass stars and substellar objects, even below the deuterium-burning mass limit, share the same formation mechanism.
\end{abstract}

Key words. stars: low mass, brown dwarfs - Galaxy: open clusters and associations: individual: $\sigma$ Orionis stars: planetary systems: protoplanetary discs

\section{Introduction}

The increasing sensitivity of photometric searches in young open clusters and star-forming regions $(1-10 \mathrm{Ma})$ has led to the direct detection of substellar objects with theoretical masses below the deuterium burning mass limit $\left(0.013 M_{\odot}\right.$ for solar metallicity; Chabrier \& Baraffe 2000). This mass limit has been used by several authors as a boundary to separate brown dwarfs from planetary-mass objects (PMOs). The first directly imaged PMOs were found as isolated objects in very young open clusters (Zapatero Osorio et al. 2000; Lucas \& Roche 2000; Najita et al. 2000). They are sometimes called isolated planetary-mass objects (IPMOs) to differentiate them from recently discovered PMOs orbiting stars and brown dwarfs (Chauvin et al. 2004, 2005; Neuhäuser et al. 2005; Caballero et al. 2006b) and from exoplanets detected via radial velocity, transit and microlensing methods (e.g. Mayor \& Queloz 1995; Charbonneau et al. 2000;

* Alexander von Humboldt Fellow at the Max-Planck-Institut für Astronomie

$\star \star$ Appendices are only available in electronic form at http://www. aanda.org
Beaulieu et al. 2006). The first spectroscopic data on IPMOs obtained by Zapatero Osorio et al. (2000) confirmed the cool atmospheres of several of these objects, which appear rather similar to those of PMOs orbiting stars. The origin of both IPMOs and PMOs around stars remains uncertain. It is likely that IPMOs form as a natural extension of the process that leads to the formation of low-mass stars and, probably, brown dwarfs, but they could also originate in protoplanetary discs and be ejected through dynamical interactions (Boss 2000; Reipurth \& Clarke 2001; Bate et al. 2002). It has been postulated that there is a minimum mass for formation of objects via fragmentation in molecular clouds, the so-called opacity mass-limit (Rees 1976; Silk 1977; Tohline 1980; Bate et al. 2003). This theoretical limit is expected to be in the range $0.010-0.005 M_{\odot}$. It is therefore crucial to extend current surveys in order to identify objects with masses as low as possible. The behaviour of the mass function at such low masses will be valuable to establish the formation mechanism of the IPMOs.

At higher masses than in the planetary domain, the mass spectrum gives important hints on how stars and brown dwarfs form in molecular clouds (e.g. Kroupa 2001). Following the 
Table 1. Basic log of the $I$ - and $J$-band runs.

\begin{tabular}{ccccccccc}
\hline \hline Telescope & Instrument & Filter & Dates & $\begin{array}{c}\text { Exp. time } \\
(\mathrm{s})\end{array}$ & $\begin{array}{c}\text { Area } \\
\left(\operatorname{arcmin}^{2}\right)\end{array}$ & $\begin{array}{c}F W H M \\
(\operatorname{arcsec})\end{array}$ & $\begin{array}{c}\text { Compl. } \\
(\mathrm{mag})\end{array}$ & $\begin{array}{c}\text { Limit. } \\
(\mathrm{mag})\end{array}$ \\
\hline INT & WFC & RGO I & 2000 Dec. 30-2001 Jan. 1 (WFC00) & $21 \times 1500$ & 970 & $1.2-1.3$ & 23.4 & 24.0 \\
& & & 2003 Jan. 8 (WFC03) & $20 \times 1200$ & 970 & $1.0-1.1$ & 23.3 & 24.1 \\
\multirow{2}{*}{ Antu } & \multirow{2}{*}{ ISAAC } & \multirow{2}{*}{} & 2001 Dec. 8,9,10,18 & $4 \times 60$ & 680 & $0.4-0.7$ & 20.6 & 21.8 \\
& & & 2001 Dec. 8,9,10,18 & $2 \times 60^{a}$ & 110 & $0.4-0.7$ & 20.2 & 21.4 \\
\hline
\end{tabular}

$\left.{ }^{a}\right]$ These are areas along the borders of the ISAAC scans and have shorter integration times.

nomenclature of Scalo (1986), the mass spectrum can be approximated by a power-law function $\Delta N / \Delta M \approx a M^{-\alpha}$ (where $\alpha=-\gamma$ and $\Delta N$ is the number of objects in the mass interval $\Delta \mathrm{M})$. The substellar mass function in the brown dwarf domain has been investigated in several open clusters, from the very nearby relatively old Hyades cluster to much younger and distant star-forming regions (see e.g. Zapatero Osorio et al. 1997; Bouvier et al. 1998; Luhman 2000; Barrado y Navascués et al. 2004; Bihain et al. 2006, and references therein). These studies point out a decrease of the slope in the power law at low mass. However, the deepest searches suggest that the mass spectrum is still rising even below the deuterium burning mass limit (Béjar et al. 2001; Muench et al. 2002; Greaves et al. 2003; Oasa et al. 2006; González-García et al. 2006).

The $\sigma$ Orionis cluster has some advantages as a place for searching for and characterizing substellar objects. In particular, it is nearby and very young. Here we adopt an age of $3 \pm 2 \mathrm{Ma}$ (Oliveira et al. 2002; Zapatero Osorio et al. 2002a; Sherry et al. 2004) and a heliocentric distance of $360_{-60}^{+70} \mathrm{pc}$ (Brown et al. 1994). The cluster is relatively free of extinction $\left(A_{\mathrm{V}}<1 \mathrm{mag}\right.$; Lee 1968; Béjar et al. 2004) and has a moderate spatial member density, solar composition $([\mathrm{Fe} / \mathrm{H}]=0.0 \pm 0.1$; Caballero 2005) and a large frequency of intermediate-mass stars with discs (33 $\pm 6 \%$; Oliveira et al. 2006). A compilation of different determinations of the age, distance and disc frequency at different mass intervals is provided in Caballero (2007). The cluster contains several dozen brown dwarfs with spectroscopic features of youth and with discs (Zapatero Osorio et al. 2002a; Barrado y Navascués et al. 2002a; Muzerolle et al. 2003; Kenyon et al. 2005; Caballero et al. 2006a). It is also the star forming region with the largest number of candidate IPMOs with followup spectroscopy (Zapatero Osorio et al. 2000, 2002b,c; Martín et al. 2001; Barrado y Navascués et al. 2001; Martín \& Zapatero Osorio 2003).

Our present study is a natural extension of the work of Béjar et al. (2001), who presented the first substellar mass function in the $\sigma$ Orionis cluster. Here we investigate the mass function down to a few Jupiter masses and use recent data from 1 to $8 \mu \mathrm{m}$ that provide information on the existence of circumsubstellar discs.

\section{The optical-near infrared search}

We used the Wide Field Camera (WFC) at the 2.5-m Isaac Newton Telescope (INT) and the Hawaii arm (SW) of ISAAC at the 8.2-m Very Large Telescope (VLT) UT1 Antu to obtain very deep $I$ - and $J$-band imaging. We studied a $0.22-\mathrm{deg}^{2}$ region to the southeast of the OB quintuple star system $\sigma$ Ori that gives the name to the $\sigma$ Orionis cluster. Table 1 lists the basic data of both optical and near-infrared campaigns (dates, exposure times, survey area and average seeing).
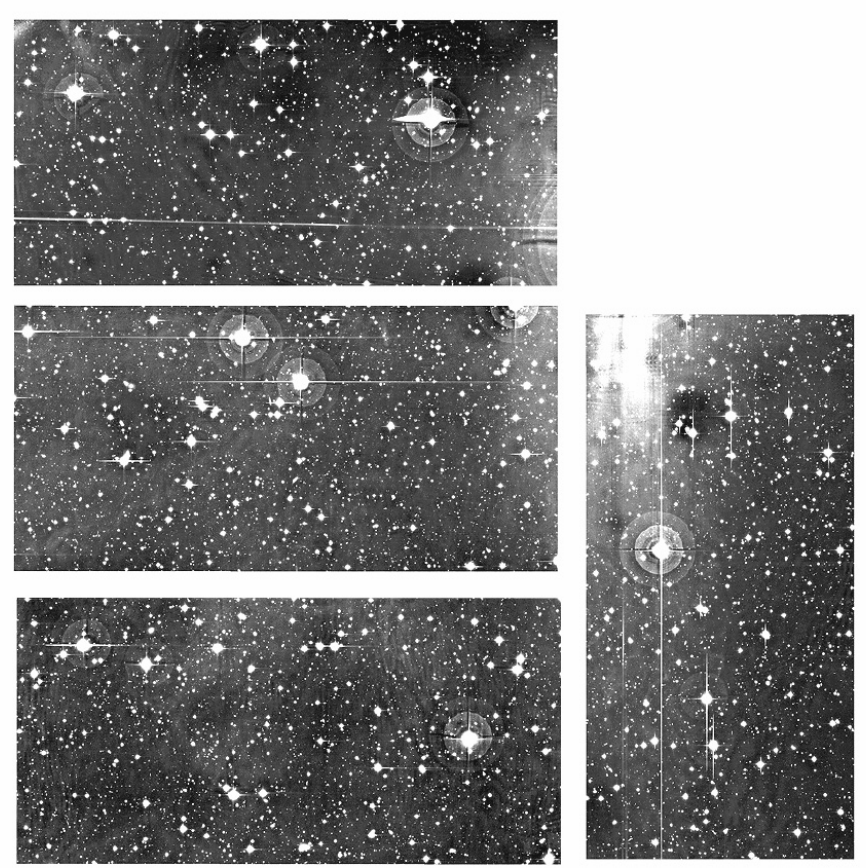

Fig. 1. Mosaic of the $I$-band images taken with WFC/INT (WFC03). North is up and east to the left. The size of each WFC chip is about 11 $\times 22 \operatorname{arcmin}^{2}$. The glare of the OB star system $\sigma$ Ori is clearly visible to the northeast of the vertical detector (CCD\#2). The mosaic on to a DSS-2-IR image centred on $\sigma$ Ori can be found in Fig. 4 of Caballero (2005). See also the false-colour composite image in Fig. B.1, available on-line.

The central coordinates of the survey (05:39:39 - 02:44:40 J2000) were chosen to take advantage of the asymmetric configuration of the four WFC chips. Some of the brightest cluster stars, especially those in the central $\sigma$ Ori system as well as HD $37525 \mathrm{AB}$, were placed out of the field of view or in the gaps between detectors. This configuration was a compromise to study both the maximum area close to the cluster centre - where the spatial density of cluster members is larger - and the minimum area affected by the glare of bright stars (see Fig. 1). The survey area is quite far from the location of the bulk of objects of the group 1 in Jeffries et al. (2006), which have an average radial velocity different from that adopted for the $\sigma$ Orionis cluster (see also Caballero 2007).

\subsection{I-band data}

Two different optical datasets were obtained with the WFC/INT $\left(4 \times 4 \mathrm{k} \times 2 \mathrm{k}, 0.333 \operatorname{arcsec} \mathrm{pix}^{-1}\right)$ and the RGO I filter (see Table 1). Both datasets, named WFC00 and WFC03 after the 
years of observation (2000 and 2003), roughly share the same depth and coordinates of the centre of field. The older dataset (WFC00), however, was taken with a $25 \%$ longer exposure time, leading to strong artefacts surrounding bright stars. The seeing conditions were also slightly worse, which made the combination of the two datasets impractical. The night of 2003 Jan. 8 (WFC03), during which we imaged several Landolt (1992) standard stars in the $I$-band, was photometric.

We reduced the data using standard procedures including bias subtraction and flat fielding, and performed aperture and PSF photometry using noao.imred and noao.digiphot.daophot routines within the IRAF environment, as described in Caballero et al. (2004). About 30000 optical sources were detected in each dataset. To avoid spurious detections, we discarded sources with very large errors in their instrumental magnitudes $(0.2$ and $0.5 \mathrm{mag}$ for $I<20$ and $I>20$ mag respectively) leaving $\sim 25000$ reliable optical sources for each dataset. The total covered field of view for each dataset was $970 \mathrm{arcmin}^{2}$. However, 3-4\% (WFC00) and $1-2 \%$ (WFC03) of the area was useless due to bleeding lines by saturated bright stars, their surrounding glares or incomplete correction of chip dead columns during flat fielding.

Both datasets have also been used to study the photometric variability of S Ori 45 (Zapatero Osorio et al. 2003) and another 27 young brown dwarfs of the $\sigma$ Orionis cluster (Caballero et al. 2004).

\subsection{J-band data}

We obtained 27 data blocks with ISAAC/VLT $(1 \mathrm{k} \times 2 \mathrm{k}$, $\left.0.148 \operatorname{arcsec}^{-1}\right)^{-1}$ ) and the $J$ filter during three consecutive nights in Dec. 2001. Two more data blocks were obtained in service mode one week later (2001 Dec 18; three $J$-band data blocks were missing to cover the $I$-band survey completely). The sky was free of clouds except for the first night, which was partially covered by thin cirrus. The seeing was excellent during all the nights (FWHM as low as 0.5 arcsec). Dark- and skysubtraction, flat fielding and alignment and combination of the data blocks were performed with IRAF. The overlapping area between optical (WFC) and near-infrared (ISAAC) images was $790 \operatorname{arcmin}^{2}$.

For easier handling, the individual $J$-band frames were aligned and combined in long scans, with sizes up to 22.0 $\times 2.4 \mathrm{arcmin}^{2}$. Every region in the survey area was observed for $240 \mathrm{~s}$ except for the borders of the scans $\left(1.2 \times 2.4 \mathrm{arcmin}^{2}\right.$ on each side), which received half the integration time.

Near-infrared point sources were detected using noao.digiphot.daophot.daofind. Aperture and PSF photometries were performed using other routines of the noao.digiphot.daophot package within the IRAF environment, in the same way as in the optical. We detected 11500 $J$-band sources.

\subsection{Photometric calibration and completeness}

We calibrated our $J$-band photometry to the Two-Micron All Sky Survey Catalogue system (2MASS; Cutri et al. 2003) using selected sources in common between our ISAAC images and the 2MASS catalogue. The useful overlapping magnitude interval between our deep images and the 2MASS data lies in the narrow interval $14.5 \mathrm{mag} \leq J \leq 15.5 \mathrm{mag}$. Hence, we could only use about a dozen comparison stars in each ISAAC scan to establish the zero-point calibration for our near-infrared data. This leads to calibration uncertainties twice as large as the typical 2MASS errors. The standard deviation in the $J$ calibrations was in general less than 0.09 mag.

The photometric calibration in the optical using photometric standard stars from Landolt (1992) observed at different zenith distances during the WFC03 run provided accuracies better than 0.05 mag. We observed only in the I-band filter so no colourdependent term could be used in the calibration. As the Landolt standards are not very red, the photometry for the fainter (redder) objects might suffer from unknown systematic effects. Each of the four WFC chips was calibrated independently. The WFC00 dataset was calibrated using bright WFC03 sources in common as a reference. Except for intrinsically variable cluster members and for very faint optical sources with large Poisson photon errors, the agreement between the photometry of both WFC00 and WFC03 datasets is of the order of the photometric calibration uncertainty (see further details in Caballero et al. 2004). We also compared the photometric data of objects with magnitudes fainter than $I=16.5 \mathrm{mag}$ in common with other independent surveys in the area (the photometry of brighter ones may be affected by non-linear or saturation effects in our WFC images). Within the uncertainties, there is no appreciable difference between the I-band magnitudes in our study and those of Béjar et al. (1999, 2004). However, Kenyon et al. (2005), based on Sloan Gunn $i^{\prime}$ - and Harris $R$-band photometry, provided $I$-band magnitudes for objects in common with our survey that are almost 0.3 mag fainter. This difference in magnitudes is probably due to the different filter systems used and/or the absence of colour-dependent terms in our photometric calibration. The photometry from Béjar et al. (2001) must not be used for comparison, since it has been found to be incorrect. The photometry from this work has been re-calibrated, using data taken during the same night as the one we are using to calibrate our I-band survey, and some systematic errors due to variation of the zero-points between the WFC chips have been found (V. J. S. Béjar et al., in prep.).

Completeness and limiting magnitudes in the $I$ - and $J$-bands are shown in the last columns of Table 1. As completeness we take the point at which the number of detected sources per magnitude interval stops increasing with a fixed power law of the magnitude, $N(m) \propto m^{p}$ (where $p$ is an arbitrary real number). The measured number of sources departures $50 \%$ from the power law at the limiting magnitude. Our completeness and limiting magnitudes are roughly equivalent to the $10 \sigma$ and $3 \sigma$ mag, respectively. The shallower borders along the ISAAC scans led to brighter completeness and detection limits by $2.5 \log 2^{1 / 2} \sim$ $0.4 \mathrm{mag}$.

Given the completeness limits of the $I$ and $J$ surveys and the expected colours of the objects of interest, the I-band photometry sets the limiting magnitude. Our faintest cluster member candidates are however brighter than the completeness limits in $I$ and $J$ (see Sect. 3.2). The optical data also sets the bright limit: the confirmed stellar cluster member S Ori 8, with $J=14.14 \pm 0.03 \mathrm{mag}$ and $I=15.74 \pm 0.04 \mathrm{mag}$ (2MASS Catalogue and Béjar et al. 1999), was detected in the non-linear regime of the near-infrared images, but saturated in the optical images. It is the only known cluster member in the area fainter than $J=14.0$ mag that has not been studied here.

\subsection{Astrometric calibration and combination of I- and J-band data}

We used the USNO-A2 and 2MASS astro-photometric catalogues to transform from the physical coordinates of the WFC chips and the ISAAC scans to celestial coordinates (for further 
details see Bihain et al. 2006). The accuracies in these astrometric solutions were about 0.1 and 0.2 arcsec for the $I$-band images and the $J$-band scans, respectively (about twice the average catalogue errors).

After cross matching positions between near-infrared and optical sources, we derived mean coordinates and $I$ - and $J$-band magnitudes for $\sim 9400$ objects. About 2100 near-infrared sources were not correlated with any optical source. Among them, a few hundred objects within the completeness of the $J$-band images and without optical counterparts (i.e. with expected red $I-J$ colours) were visually inspected on the WFC images, since they could be very low-mass cluster members fainter than our adopted completeness limit in the optical data. After this analysis, we concluded that the vast majority were not detected in the I-band images because they were: $(i)$ double or multiple background stars or galactic nuclei only resolved in the ISAAC data; (ii) faint sources in the glare of bright stars in the optical; (iii) artefacts in the ISAAC frames; (iv) multiple detections of saturated bright stars or $(v)$ extended sources of extragalactic nature. Some promising near-infrared sources without optical counterpart were followed up with deep $H K$ imaging (see Sect. 3.3.2).

\section{Cluster member selection and the infrared follow-up}

\subsection{Known objects in the survey area}

In the studied area and magnitude intervals (16.1 mag $\lesssim$ $I \lesssim 24.1 \mathrm{mag}$ and $14.2 \mathrm{mag} \lesssim J \lesssim 20.6 \mathrm{mag}$ ) there are 50 cluster members and cluster member candidates reported in the literature. They have been studied in the optical surveys by: Béjar et al. (1999 - Bé99 -, 2001, 2004); Zapatero Osorio et al. (2000); Caballero et al. (2004 - Ca04 -); Kenyon et al. (2005 - Ke05 -); González-García et al. (2006), and in specific works such as: Zapatero Osorio et al. (1999, 2002a - ZO02 -); Barrado y Navascués et al. (2002a - ByN02 -, 2003 - ByN03 -); Muzerolle et al. (2003 - Mu03 -); McGovern et al. (2004 - McG04 -); Scholz \& Eislöffel (2004); Burningham et al. (2005a - Bu05-); Caballero et al. (2006a - Ca06 -); Franciosini et al. (2006Fr06-).

Out of these 50 objects, only one, S Ori 69 , was not detected on our images. This non-detection suggests that the isolated planetary-mass object candidate is fainter than what was published in the literature $(I=23.9 \pm 0.2 \mathrm{mag}, J=20.2 \pm 0.4 \mathrm{mag}$; Zapatero Osorio et al. 2000; Martín et al. 2001). G. Bihain et al. (private comm.) have recently obtained new deep near-infrared imaging of S Ori 69, determining its $J$-band magnitude at 21.61 $\pm 0.16 \mathrm{mag}$. Two out of the 49 identified targets in the area, S Ori 41 and S Ori J054004.9-024656, were classified as probable non-members by Béjar et al. $(2001,2004)$ (the latter is a visual binary resolved in our ISAAC images, with $\rho \lesssim 0.8 \operatorname{arcsec}$ ). Also, the M5.0 \pm 0.5-type dwarf S Ori J053909.9-022814 does not display signatures of youth in high-quality optical spectra (Barrado y Navascués et al. 2003; Kenyon et al. 2005). We do not consider these three objects as cluster members.

There remain 46 cluster members and candidates reported in the literature in the survey area. Spectroscopic information is available for 31 of them. For 11 of these objects, only spectral types could be determined, while the other 20 members display spectroscopic features of extreme youth (age $<10 \mathrm{Ma}$ ). Specifically, these features are: Li I $16707.8 \AA$ in absorption, broad and/or strong $\mathrm{H} \alpha$ emission, weak alkali absorption lines (i.e. low-gravity, normally measured from the $\mathrm{pEW}(\mathrm{Na} \mathrm{I})$ in

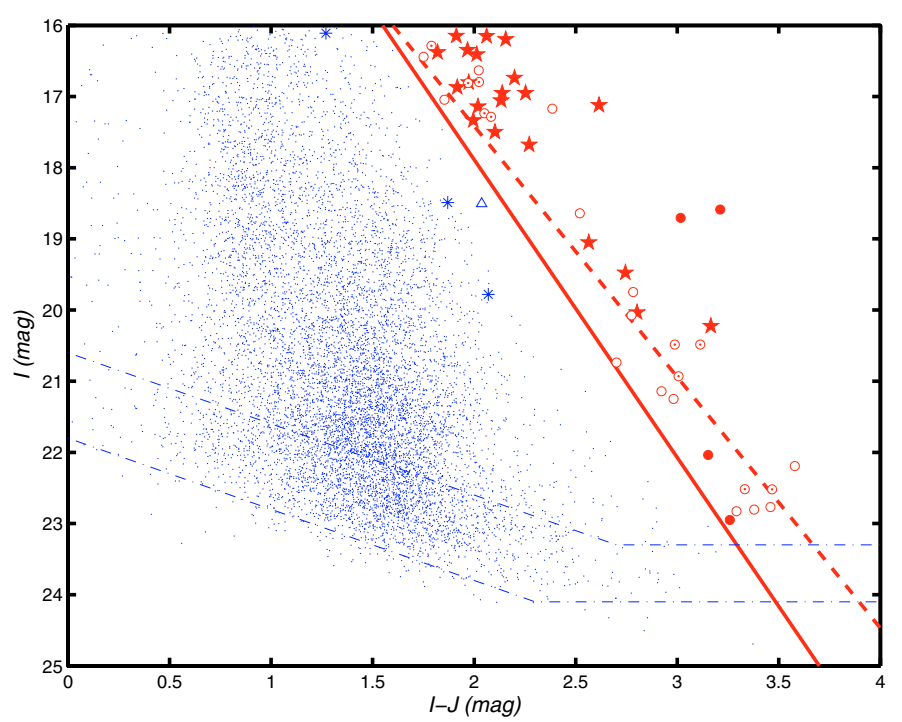

Fig. $2 . ~ I$ vs. $I-J$ colour-magnitude diagram from our WFC03/INTISAAC/Antu survey. The different symbols indicate: probable foreand background sources (small dots, "."); spectroscopically confirmed cluster members from Table 2 (filled stars, " $\star$ "); cluster member candidates with spectral type determination (encircled dots, "๑"); cluster member candidates without spectral type determination (open circles, "o"); the four new cluster member candidates (filled circles, "•"); S Ori J053948.1-022914 (the open triangle, “ $\Delta ")$; the three detected cluster non-members (asterisks, "*”). Dash-dotted lines indicate the completeness and detection limits of our survey. The dashed line is the lower envelope of confirmed cluster members with youth indicators. The solid line is the boundary considered as the selection criterion. Colour versions of all our figures are available in the electronic publication.

optical spectra, except in the case of S Ori 51 where a $J$-band spectrum was used), and emission lines ascribed to accretion processes or outflows (e.g. [O I] $\lambda 6300.3 \AA$, He I $\lambda 5875.8 \AA$ ). Most of them have radial velocities, $V_{r}$, similar to the cluster systemic radial velocity of $30-35 \mathrm{~km} \mathrm{~s}^{-1}$. Additionally, some of them display optical photometric variability with an amplitude larger than $0.07 \mathrm{mag}$ ("var."), $K_{\mathrm{s}}$-band flux excess ("nIR"), or $\mathrm{X}$-ray emission ("X"). There is insufficient and/or discrepant membership information on S Ori 20 (Barrado y Navascués et al. 2003; Kenyon et al. 2005), S Ori 47 (Zapatero Osorio et al. 1999; McGovern et al. 2004), and S Ori J053844.4-024037 (Burningham et al. 2005a). We do not consider these three objects as spectroscopically confirmed young cluster members. The 20 confirmed cluster members together with their references and most important characteristics are given in Table 2.

\subsection{Cluster member selection from the I vs. I - J diagram}

We have selected the cluster member candidates from our WFC0 3 + ISAAC data, using the $I$ vs. $I-J$ colour-magnitude diagram shown in Fig. 2. Among the 9400 sources with optical and near-infrared information in our survey, we have selected new photometric candidate cluster members based on their position in the diagram with respect to the 20 spectroscopically confirmed cluster members, indicated by filled stars. The latter define the spectrophotometric sequence of the $\sigma$ Orionis cluster; this is redder than the field stars. Most of them are brighter than $I=18.0 \mathrm{mag}$, while only four brown dwarfs with spectroscopic features indicative of youth fall in the interval $19.0 \leq I \leq$ 20.5 mag. Five brown dwarf and IPMO candidates fainter than 
Table 2. Objects with signatures of extreme youth and cluster membership in the survey area compiled from the literature.

\begin{tabular}{|c|c|c|c|c|c|c|}
\hline Name & $\begin{array}{c}\mathrm{p} E W(\operatorname{Li~I}) \\
(\AA)\end{array}$ & $\begin{array}{c}\mathrm{p} E W(\mathrm{H} \alpha) \\
(\AA)\end{array}$ & low $g^{a}$ & $V_{r}^{b}$ & Remarks & References \\
\hline S Ori J054000.2-025159 & $+0.25 \pm 0.09$ & & yes & bin. & & $\mathrm{Ke} 05$ \\
\hline S Ori J053848.1-024401 & $+0.53 \pm 0.10$ & & yes & yes & & $\mathrm{Ke} 05$ \\
\hline S Ori J053833.9-024508 & $+0.38 \pm 0.07$ & & yes & yes & & $\mathrm{Ke} 05$ \\
\hline S Ori J053911.4-023333 & $+0.33 \pm 0.06$ & $-4.7 \pm 1.0$ & yes & yes & & ByN03, Ke05 \\
\hline S Ori 14 & $+0.71 \pm 0.06$ & & yes & yes & & $\mathrm{Ke} 05$ \\
\hline S Ori J054014.0-023127 & $+0.47 \pm 0.08$ & broad & yes & yes & var. & $\mathrm{Ca} 04, \mathrm{Ke} 05$ \\
\hline S Ori J053847.2-025756 & $+0.53 \pm 0.10$ & broad & yes & yes & var. & $\mathrm{Ca} 04, \mathrm{Ke} 05$ \\
\hline S Ori J053838.6-024157 & $+0.55 \pm 0.06$ & $-6 \pm 1$ & yes & yes & & $\mathrm{Ke} 05, \mathrm{Bu} 05, \mathrm{Ca} 06$ \\
\hline S Ori 25 & $+0.53 \pm 0.10$ & $-45.0 \pm 1.0$ & yes & yes & var., He I, X & Bé99, Mu03, Fr06 \\
\hline S Ori J053826.1-024041 & $+0.51 \pm 0.08$ & $-4 \pm 2$ & yes & yes & & ByN03, Ke05 \\
\hline S Ori 27 & $+0.74 \pm 0.09$ & $-5.7 \pm 1.5$ & yes & yes & & $\mathrm{ZO} 02, \mathrm{Ke} 05$ \\
\hline S Ori J053825.4-024241 & & $-260 \pm 30$ & & yes & var., nIR, He I & $\mathrm{Ca} 06$ \\
\hline S Ori 28 & $+0.66 \pm 0.09$ & & yes & yes & & $\mathrm{Ke} 05$ \\
\hline S Ori 32 & $+0.46 \pm 0.09$ & & yes & yes & & $\mathrm{Ke} 05$ \\
\hline S Ori J054004.5-023642 & $<0.39$ & broad & yes & yes & var. & $\mathrm{Ke} 05$ \\
\hline S Ori 36 & $+0.45 \pm 0.15$ & & yes & bin. & & $\mathrm{Ke} 05$ \\
\hline S Ori 42 & & $-89 \pm 12$ & & & var. & ByN03, Ca04 \\
\hline S Ori 45 & $+2.4 \pm 1.0$ & {$[-60,-20]$} & yes & bin.? & & Bé99, ZO02 \\
\hline S Ori 51 & & $-25:$ & $\operatorname{yes}(J)$ & & & ByN03, McG04 \\
\hline S Ori 71 & & $-700 \pm 80$ & & & var.? & Ca04, ByN02 \\
\hline
\end{tabular}

a Low-gravity features.

$b$ Radial velocity consistent with membership in cluster. Symbol "bin." denotes possible binary according to Kenyon et al. (2005).

$I=19.0 \mathrm{mag}$ have low-resolution spectroscopy available in the literature and extrapolate the spectrophotometric cluster sequence towards fainter magnitudes and lower masses. The lower envelope of the confirmed candidates from $I=16.0$ to $20.5 \mathrm{mag}$ is indicated by a dashed line in the diagram. Our adopted selection criterion for cluster membership (solid line in Fig. 2) is the lower envelope of cluster members shifted to the blue to accommodate photometric uncertainties (by $0.05 \mathrm{mag}$ at $I=16 \mathrm{mag}$ and $0.45 \mathrm{mag}$ at $I=25 \mathrm{mag}$ ). There are 49 objects ( 45 previously known) to the right of this boundary, which is the final cluster list we will use throughout the present paper. All previously reported $\sigma$ Orionis candidates which are located within our survey area and magnitude limits lie to the right of the membership boundary. The only exception is S Ori J053948.1-022914, the properties of which are summarised in Sect. A.1. Identifications, J2000 coordinates, WFC03/INT $I$-band and ISAAC/VLT $J$-band magnitudes and spectral types when available of the 49 selected targets are detailed in Table 3. The spatial distribution of the $49 \sigma$ Orionis members and member candidates is shown in Fig. 3.

Of the 49 objects, four are newly identified in our survey. For their identifications we follow the nomenclature of Béjar et al. (2001): S Ori Jhhmmss.s-ddmmss. These "S Ori" sources are not, however, associated with the variable star S Ori (HD 36090), which is located several degrees away. The two brightest new objects are red candidate brown dwarfs that were embedded in the glare of $\sigma$ Ori in the northwest corner of CCD\#4 (in the centre of the WFC00 mosaic) in Caballero et al. (2004). The other two new objects, with $I=22-23 \mathrm{mag}$, fell in the $I$ vs. $I-J$ diagram slightly to the left of the $10 \mathrm{Ma}$-old Dusty00 isochrone (Chabrier et al. 2000), which was used by Caballero et al. (2004) to differentiate candidate cluster members from probable foreground and background sources.

Six bright sources in our final sample were saturated in the longer-exposure WFC00 images, too. All of our $\sigma$ Orionis members and member candidates have been selected from the combination of the WFC03 optical dataset and the ISAAC $J$-band images. The combination of the WFC00 optical dataset and the ISAAC data yields identical results in the magnitude interval common to the two epochs of WFC observations. The exceptions are the two candidate brown dwarfs whose photometry was contaminated by the glare of $\sigma$ Ori in the WFC00 data and the six stars saturated in the longer WFC00 exposures. This supports our selection of cluster candidates and provides evidence for the fact that photometric optical variability does not significantly affect our selection criterion.

\subsection{The infrared follow-up}

In oder to identify foreground contaminants we obtained infrared data from the IRAC/Spitzer Space Telescope archive and from 2MASS and performed additional deep $H$ - and $K_{\mathrm{s}}$ imaging.

\subsubsection{IRAC/Spitzer follow-up}

We used the Infrared Array Camera (IRAC) deep image mosaics centred on $\sigma$ Ori from the Spitzer Space Telescope Archive in the $3.6,4.5,5.8$, and $8.0 \mu \mathrm{m}$ bands (denoted here as [3.6], [4.5], [5.8], and [8.0]). We extracted post-basic calibrated data with the Leopard tool, performed 5-arcsec standard aperture photometry with IRAF on sources with $\mathrm{S} / \mathrm{N}$ ratio $>3$, applied the corresponding aperture correction factors, and converted the measured flux per pixel into calibrated magnitudes in the Vega system using the zero-points for each channel of the IRAC instrument. We performed additional PSF photometry of some of the faintest sources. Neither array-location-dependent nor colour corrections were applied. Up-to-date information on the photometry and absolute calibration of IRAC data is given at http://ssc.spitzer. caltech. edu/irac. The completeness magnitudes of the mosaic images in the [3.6], [4.5], [5.8], and [8.0] bands are 17.2,17.2, 15.0, and 14.0 mag, respectively, with uncertainties of $0.3 \mathrm{mag}$. Our magnitudes deviate no more 
Table 3. Basic data of our $\sigma$ Orionis cluster members and candidates.

\begin{tabular}{|c|c|c|c|c|c|c|c|c|}
\hline Name & $\alpha^{J 2000}$ & $\delta^{J 2000}$ & $I$ (mag) & $J$ (mag) & $\log \frac{L}{L_{\odot}}$ & Mass $\left(M_{\odot}\right)$ & Sp.Type & Remarks $^{a}$ \\
\hline S Ori J054000.2-025159 & 054000.15 & -025159.4 & $16.19 \pm 0.04$ & $14.04 \pm 0.08$ & $-1.43 \pm 0.18$ & $0.11_{-0.05}^{+0.10}$ & & $\mathrm{Y}$ \\
\hline S Ori J053848.1-024401 & 053848.19 & -024400.8 & $16.15 \pm 0.03$ & $14.09 \pm 0.08$ & $-1.45 \pm 0.18$ & $0.10_{-0.05}^{+0.09}$ & & Y,D \\
\hline S Ori J053833.9-024508 & 053833.88 & -024507.8 & $16.15 \pm 0.03$ & $14.24 \pm 0.07$ & $-1.51 \pm 0.18$ & $0.09_{-0.04}^{+0.09}$ & & $\mathrm{Y}, \mathrm{D}$ \\
\hline S Ori J053911.4-023333 & 053911.40 & -023332.8 & $16.35 \pm 0.04$ & $14.38 \pm 0.11$ & $-1.57 \pm 0.18$ & $0.078_{-0.03}^{+0.04}$ & M5.0 \pm 0.5 & $\mathrm{Y}$ \\
\hline S Ori 14 & 053937.60 & -024430.5 & $16.41 \pm 0.04$ & $14.40 \pm 0.08$ & $-1.58 \pm 0.18$ & $0.076_{-0.03}^{+0.03}$ & & $\mathrm{Y}$ \\
\hline S Ori 11 & 053944.33 & -023302.8 & $16.29 \pm 0.04$ & $14.50 \pm 0.12$ & $-1.60 \pm 0.18$ & $0.071_{-0.02}^{+0.05}$ & M6.0 \pm 0.5 & \\
\hline S Ori J054014.0-023127 & 054013.96 & -023127.4 & $17.12 \pm 0.03$ & $14.51 \pm 0.10$ & $-1.60 \pm 0.18$ & $0.071_{-0.02}^{+0.82}$ & & Y \\
\hline S Ori J053847.2-025756 & 053847.15 & -025755.7 & $16.74 \pm 0.02$ & $14.54 \pm 0.08$ & $-1.63 \pm 0.18$ & $0.066_{-0.02}^{+0.02}$ & & Y,D \\
\hline S Ori J053838.6-024157 & 053838.59 & -024155.9 & $16.38 \pm 0.03$ & $14.56 \pm 0.07$ & $-1.63 \pm 0.18$ & $0.067_{-0.02}^{+0.02}$ & M5.5 \pm 1.0 & $\mathrm{Y}$ \\
\hline S Ori 16 & 053915.10 & -024047.6 & $16.63 \pm 0.03$ & $14.61 \pm 0.10$ & $-1.66 \pm 0.18$ & $0.062_{-0.018}^{+0.02}$ & & \\
\hline S Ori J053902.1-023501 & 053901.94 & -023502.9 & $16.44 \pm 0.03$ & $14.69 \pm 0.11$ & $-1.67 \pm 0.18$ & $0.061_{-0.017}^{+0.018}$ & & $\mathrm{D}$ \\
\hline S Ori 25 & 053908.95 & -023958.0 & $16.95 \pm 0.03$ & $14.70 \pm 0.10$ & $-1.70 \pm 0.18$ & $0.058_{-0.016}^{+0.017}$ & M6.5 \pm 0.5 & $\mathrm{Y}$ \\
\hline S Ori J053954.3-023719 & 053954.33 & -023718.9 & $16.79 \pm 0.03$ & $14.77 \pm 0.07$ & $-1.72 \pm 0.18$ & $0.056_{-0.015}^{+0.016}$ & $\mathrm{M} 6.0 \pm 1.0$ & (D) \\
\hline S Ori J053844.4-024037 & 053844.48 & -024037.6 & $17.17 \pm 0.03$ & $14.78 \pm 0.08$ & $-1.72 \pm 0.18$ & $0.056_{-0.015}^{+0.015}$ & & $\mathrm{D}$ \\
\hline S Ori J053826.1-024041 & 053826.23 & -024041.4 & $16.95 \pm 0.02$ & $14.81 \pm 0.07$ & $-1.74 \pm 0.18$ & $0.054_{-0.014}^{+0.015}$ & M5.0 \pm 2.0 & Y \\
\hline S Ori 20 & 053907.58 & -022905.6 & $16.80 \pm 0.03$ & $14.83 \pm 0.10$ & $-1.75 \pm 0.18$ & $0.054_{-0.014}^{+0.014}$ & M5.5 \pm 0.5 & \\
\hline S Ori J053829.0-024847 & 053828.97 & -024847.3 & $16.81 \pm 0.02$ & $14.84 \pm 0.09$ & $-1.75 \pm 0.18$ & $0.053_{-0.014}^{+0.014}$ & $\mathrm{M} 6.0 \pm 0.5$ & $\mathrm{D}$ \\
\hline S Ori 27 & 053817.42 & -024024.3 & $17.05 \pm 0.03$ & $14.92 \pm 0.08$ & $-1.79 \pm 0.18$ & $0.050_{-0.013}^{+0.814}$ & M6.5 \pm 0.5 & Y \\
\hline S Ori J053825.4-024241 & 053825.44 & -024241.3 & $16.87 \pm 0.02$ & $14.95 \pm 0.07$ & $-1.79 \pm 0.18$ & $\begin{array}{l}0.050_{-0.013}^{+0.06} \\
0.050_{-0.012}\end{array}$ & $\mathrm{M} 6.0 \pm 1.0$ & Y,D \\
\hline S Ori 28 & 053923.19 & -024655.8 & $17.14 \pm 0.03$ & $15.12 \pm 0.16$ & $-1.86 \pm 0.18$ & $0.046_{-0012}^{+0.012}$ & & Y \\
\hline S Ori 31 & 053820.88 & -024613.3 & $17.23 \pm 0.02$ & $15.18 \pm 0.09$ & $-1.89 \pm 0.18$ & $0.044_{-0.011}^{+0.812}$ & $\mathrm{M} 7.0 \pm 0.5$ & \\
\hline S Ori J053922.2-024552 & 053922.25 & -024552.4 & $17.04 \pm 0.03$ & $15.20 \pm 0.15$ & $-1.89 \pm 0.18$ & $0.044_{-0.011}^{+0.011}$ & & \\
\hline S Ori 30 & 053913.08 & -023750.9 & $17.29 \pm 0.03$ & $15.20 \pm 0.07$ & $-1.90 \pm 0.18$ & $0.044_{-0.011}^{+0.811}$ & $\mathrm{M} 6.0 \pm 0.5$ & $\mathrm{D}$ \\
\hline S Ori 32 & 053943.59 & -024731.8 & $17.33 \pm 0.03$ & $15.34 \pm 0.05$ & $-1.95 \pm 0.18$ & $0.041_{-0.011}^{+0.811}$ & $1010.0 \pm 0.5$ & Y \\
\hline S Ori J053855.4-024121 & 053855.42 & -024120.8 & $18.59 \pm 0.04$ & $15.38 \pm 0.10$ & $-1.88 \pm 0.18$ & $0.045_{-0.011}^{+0.011}$ & & $\mathrm{D}, \mathrm{New}$ \\
\hline S Ori J054004.5-023642 & 054004.54 & -023642.1 & $17.50 \pm 0.03$ & $15.39 \pm 0.07$ & $-1.97 \pm 0.18$ & $0.039^{+0.041}$ & & Y,D \\
\hline S Ori 36 & 053926.85 & -023656.2 & $17.68 \pm 0.03$ & $15.40 \pm 0.07$ & $-1.97 \pm 0.18$ & $0.039_{-0.010}^{+0.810}$ & & Y,D \\
\hline S Ori J053854.9-024034 & 053854.92 & -024033.8 & $18.71 \pm 0.04$ & $15.69 \pm 0.10$ & $-2.02 \pm 0.18$ & $0.037^{+0.03}$ & & D,New \\
\hline S Ori J053918.1-025257 & 053918.13 & -025256.3 & $18.64 \pm 0.04$ & $16.12 \pm 0.08$ & $-2.25 \pm 0.18$ & $0.028^{+0.012}$ & & $\mathrm{D}$ \\
\hline S Ori 42 & 053923.41 & -024057.6 & $19.05 \pm 0.03$ & $16.49 \pm 0.10$ & $-2.39 \pm 0.18$ & $0.025^{+0.0006}$ & $\mathrm{M} 7.5 \pm 0.5$ & Y,D \\
\hline S Ori 45 & 053825.58 & -024837.0 & $19.48 \pm 0.02$ & $16.73 \pm 0.07$ & $-2.47 \pm 0.18$ & $0.023_{-0.010}^{+0.010}$ & $\mathrm{M} 8.5 \pm 0.5$ & $\mathrm{Y}$ \\
\hline S Ori J053929.4-024636 & 053929.38 & -024636.5 & $19.75 \pm 0.03$ & $16.96 \pm 0.16$ & $-2.56 \pm 0.18$ & $0.021_{-0.010}^{+0.010}$ & & \\
\hline S Ori 51 & 053903.22 & -023020.7 & $20.22 \pm 0.03$ & $17.06 \pm 0.12$ & $-2.55 \pm 0.18$ & $0.021_{-0.010}^{+0.010}$ & M9.0 \pm 0.5 & Y \\
\hline S Ori 71 & 053900.30 & -023706.7 & $20.03 \pm 0.03$ & $17.23 \pm 0.07$ & $-2.67 \pm 0.18$ & $0.019_{-0.010}^{+0.805}$ & $\mathrm{~L} 0.0 \pm 0.5$ & Y,D \\
\hline S Ori J053849.5-024934 & 053849.59 & -024933.3 & $20.08 \pm 0.02$ & $17.30 \pm 0.08$ & $-2.70 \pm 0.18$ & $0.018_{-0.010}^{+0.005}$ & & D \\
\hline S Ori 47 & 053814.62 & -024015.4 & $20.49 \pm 0.02$ & $17.37 \pm 0.08$ & $-2.68 \pm 0.18$ & $0.019_{-0.010}^{+0.005}$ & $\mathrm{~L} 1.5 \pm 0.5$ & \\
\hline S Ori 50 & 053910.81 & -023715.1 & $20.48 \pm 0.03$ & $17.50 \pm 0.07$ & $-2.75 \pm 0.18$ & $0.017_{-0.009}^{-0.010}$ & M9.0 \pm 0.5 & \\
\hline S Ori 53 & 053825.12 & -024802.7 & $20.93 \pm 0.02$ & $17.92 \pm 0.06$ & $-2.92 \pm 0.18$ & $0.014_{-0.008}^{+0.009}$ & M9.0 \pm 0.5 & \\
\hline S Ori J053944.5-025959 & 053944.55 & -025958.9 & $20.74 \pm 0.04$ & $18.04 \pm 0.08$ & $-3.00 \pm 0.18$ & $0.013_{-0.007}^{+0.008}$ & & \\
\hline S Ori J054007.0-023604 & 054006.95 & -023604.7 & $21.14 \pm 0.03$ & $18.22 \pm 0.07$ & $-3.05 \pm 0.18$ & $0.012_{-0.007}^{+0.005}$ & & \\
\hline S Ori J053956.8-025315 & 053956.83 & -025314.4 & $21.25 \pm 0.04$ & $18.27 \pm 0.08$ & $-3.06 \pm 0.18$ & $0.012_{-0.007}^{+0.005}$ & & \\
\hline S Ori J053858.6-025228 & 053858.55 & -025226.7 & $22.19 \pm 0.05$ & $18.61 \pm 0.08$ & $-3.12 \pm 0.18$ & $0.011_{-0.006}^{+0.005}$ & & \\
\hline S Ori J053949.5-023130 & 053949.52 & -023129.8 & $22.04 \pm 0.04$ & $18.89 \pm 0.10$ & $-3.29 \pm 0.18$ & $0.008_{-0.004}^{+0.0065}$ & & New \\
\hline S Ori 60 & 053937.50 & -023041.9 & $22.52 \pm 0.05$ & $19.05 \pm 0.10$ & $-3.31 \pm 0.18$ & $0.008_{-0.004}^{+0.004}$ & $\mathrm{~L} 2.0 \pm 1.0$ & \\
\hline S Ori 62 & 053942.05 & -023032.3 & $22.52 \pm 0.05$ & $19.18 \pm 0.10$ & $-3.38 \pm 0.18$ & $0.008_{-0.004}^{+0.004}$ & $\mathrm{~L} 2.0 \pm 1.5$ & \\
\hline S Ori J053844.5-025512 & 053844.56 & -025512.4 & $22.77 \pm 0.06$ & $19.31 \pm 0.08$ & $-3.42 \pm 0.18$ & $0.007_{-0.004}^{+0.004}$ & & \\
\hline S Ori J054008.5-024551 & 054008.49 & -024550.3 & $22.80 \pm 0.07$ & $19.43 \pm 0.16$ & $-3.48 \pm 0.18$ & $0.007_{-0.004}^{+0.004}$ & & \\
\hline S Ori J053932.4-025220 & 053932.42 & -025220.3 & $22.83 \pm 0.07$ & $19.54 \pm 0.08$ & $-3.53 \pm 0.18$ & $0.007_{-0.003}^{-0.004}$ & & \\
\hline S Ori J054011.6-025135 & 054011.58 & -025134.6 & $22.95 \pm 0.07$ & $19.69 \pm 0.09$ & $-3.59 \pm 0.18$ & $0.006_{-0.003}^{+0.003}$ & & New \\
\hline
\end{tabular}

a Entries for which there is a clear spectroscopic youth indicator or evidence of disc, or are firstly identified in this work are marked by "Y", "D", and "New", respectively.

than 0.10 mag from the independently calibrated magnitudes of objects in common with Hernández et al. (2007).

Of the 49 objects in our sample, three fall out of the IRAC field of view and two, the most distant objects from the cluster centre, are only in the [4.5] and [8.0] mosaics. Table 4 summarises the available IRAC data for 46 cluster members and candidates. Typical errors of the IRAC photometry vary between $\lesssim 0.1 \mathrm{mag}$ for the brightest targets and $0.5 \mathrm{mag}$ for the faintest ones. In particular, 8 and 15 objects from the $I J$ survey are too faint to be detected in the [5.8] and [8.0] bands, respectively.

\subsubsection{Ground-based HK-band follow-up}

2MASS $J H K_{\mathrm{s}}$ photometry is available for 33 targets (the 32 brightest cluster member candidates, plus S Ori 47). Since the ISAAC images were calibrated with $2 \mathrm{MASS}$, the agreement 
Table 4. Near-infrared follow-up photometry of our $\sigma$ Orionis cluster members and candidates.

\begin{tabular}{|c|c|c|c|c|c|c|c|c|}
\hline Name & $J$ (mag) & $H$ (mag) & $K_{\mathrm{s}}(\mathrm{mag})$ & [3.6] (mag) & [4.5] (mag) & [5.8] (mag) & {$[8.0$} & Flag $^{a}$ \\
\hline S Ori J054000.2-025159 & $14.14 \pm 0.03$ & $13.47 \pm 0.02$ & $13.15 \pm 0.04$ & 08 & $12.86 \pm 0.10$ & 12.63 & 0.14 & 224 \\
\hline S Ori J053848. & .03 & & & 12.51 & 0.08 & .08 & 11.08 & 2224 \\
\hline S Ori J053833.9-024508 & $14.25 \pm 0.03$ & $13.68 \pm 0.03$ & $13.35 \pm 0.04$ & $12.81 \pm 0.08$ & $12.64 \pm 0.09$ & $12.33 \pm 0.10$ & $11.44 \pm 0.09$ & 224 \\
\hline S Ori J053911.4-023333 & $14.45 \pm 0.03$ & $13.93 \pm 0.03$ & $13.57 \pm 0.04$ & $13.28 \pm 0.10$ & $13.10 \pm 0.11$ & $12.95 \pm 0.13$ & $13.04 \pm 0.19$ & 2224 \\
\hline S Ori 14 & $14.38 \pm 0.03$ & $13.82 \pm 0.03$ & $13.38 \pm 0.03$ & $13.02 \pm 0.09$ & $12.92 \pm 0.10$ & $12.78 \pm 0.12$ & $12.83 \pm 0.17$ & 2224 \\
\hline S Ori 11 & $14.29 \pm 0.03$ & $13.72 \pm 0.03$ & $13.36 \pm 0.04$ & $13.21 \pm 0.10$ & $13.05 \pm 0.11$ & $12.92 \pm 0.13$ & $12.79 \pm 0.17$ & 2224 \\
\hline S Ori J054014.0-023127 & $14.57 \pm 0.03$ & $13.98 \pm 0.03$ & $13.57 \pm 0.04$ & - & - & - & - & 2220 \\
\hline S Ori J053847.2-025756 & $14.52 \pm 0.03$ & $13.94 \pm 0.04$ & $13.46 \pm 0.04$ & - & $12.62 \pm$ & _- & $11.42 \pm$ & 2222 \\
\hline S Ori J053838.6-024157 & $14.56 \pm 0.03$ & $13.96 \pm 0.03$ & $13.65 \pm 0.04$ & $13.33 \pm 0.10$ & $13.20=$ & $13.25 \pm 0.15$ & .21 & 2224 \\
\hline S Ori 16 & $14.67 \pm 0.03$ & $14.04 \pm 0.04$ & $13.66 \pm 0.04$ & $13.23 \pm 0.10$ & $13.55 \pm 0.12$ & $13.07=$ & $13.05=$ & 2224 \\
\hline S Ori J053902.1-023501 & $14.44 \pm 0.04$ & $13.38 \pm 0.03$ & $12.61 \pm 0.03$ & $11.45 \pm 0.04$ & $10.99 \pm 0.04$ & $10.52 \pm$ & $9.75 \pm$ & 224 \\
\hline S Ori 25 & $14.66 \pm 0.03$ & $14.14 \pm 0.04$ & $13.74 \pm 0.05$ & $13.25 \pm 0.10$ & $13.18 \pm 0.11$ & $13.22 \pm$ & $13.01 \pm$ & 2224 \\
\hline S Ori J053954.3-023719 & $14.75 \pm 0.03$ & $14.21 \pm 0.04$ & 13.80 & $13.36 \pm 0.10$ & 13.30 & $13.36 \pm$ & 13.25 & 2224 \\
\hline S Ori J053844.4-024037 & $14.80 \pm 0.03$ & $14.21 \pm 0.04$ & $13.94 \pm 0.05$ & $13.45 \pm 0.14$ & $13.06 \pm 0.11$ & $12.63 \pm 0.11$ & $12.29 \pm 0.15$ & 2224 \\
\hline S Ori J053826.1-024041 & $14.91 \pm 0.04$ & $14.28 \pm 0.04$ & 13. & $13.60 \pm 0.12$ & $13.44 \pm 0.13$ & $13.28 \pm 0.15$ & $13.17 \pm 0.20$ & 2224 \\
\hline S Ori 20 & $14.96 \pm 0.04$ & $14.34 \pm 0.04$ & $13.90 \pm 0.05$ & $13.52 \pm 0.11$ & $13.49 \pm 0.13$ & $13.24 \pm 0.15$ & $13.48 \pm 0.23$ & 2224 \\
\hline S Ori J053829.0-024847 & $14.82 \pm 0.04$ & $14.28 \pm 0.04$ & $13.88 \pm 0.06$ & $13.16 \pm 0.09$ & $12.85 \pm 0.10$ & $12.46 \pm 0.10$ & $11.82 \pm 0.10$ & 2224 \\
\hline S Ori 27 & $14.83 \pm 0.03$ & $14.31 \pm 0.04$ & $14.09 \pm 0.05$ & $13.60 \pm 0.12$ & $13.42 \pm 0.13$ & $13.54 \pm 0.17$ & $13.43 \pm 0.22$ & 2224 \\
\hline S Ori J053825.4-024241 & $14.88 \pm 0.03$ & $14.16 \pm 0.04$ & $13.57 \pm 0.03$ & $12.72 \pm 0.08$ & $12.38 \pm 0.08$ & $11.96 \pm 0.08$ & $11.26 \pm 0.08$ & 2224 \\
\hline S Ori 28 & $15.33 \pm 0.04$ & $14.78 \pm 0.04$ & $14.34 \pm 0.07$ & $13.91 \pm 0.13$ & $13.87 \pm 0.16$ & $13.80 \pm 0.20$ & $13.80 \pm 0.27$ & 2224 \\
\hline S Ori 31 & $15.19 \pm 0.04$ & $14.57 \pm 0.05$ & $14.16 \pm 0.08$ & $13.82 \pm 0.13$ & $13.63 \pm 0.14$ & & $13.51=$ & 2224 \\
\hline S Ori J053922.2-024552 & \pm 0.04 & \pm 0.05 & & 0.16 & 16 & 13. & .28 & 2224 \\
\hline S Ori 30 & 04 & 14. & 14.2 & 13. & & 13. & 14 & 224 \\
\hline S Ori 32 & 0.05 & $14 .^{\circ}$ & 14. & 13. & 16 & 13. & 13. & 224 \\
\hline S Ori J0 & 0.10 & .05 & 13. & 12.8 & .08 & 12. & .08 & 2224 \\
\hline $.5-023642$ & 0.05 & 14.8 & 14.27 & 0.12 & .12 & 12. & 12 & 2224 \\
\hline S Ori 36 & $15.46 \pm 0.04$ & $14.84 \pm 0.05$ & $14.49 \pm 0.06$ & $13.71 \pm 0.12$ & $13.48 \pm 0.13$ & $13.04 \pm 0.13$ & 0.14 & 2224 \\
\hline S Ori J053854.9-024034 & $15.92 \pm 0.06$ & $15.17 \pm 0.06$ & 14.71 & $13.79 \pm 0.13$ & 0.13 & $12.91 \pm 0.13$ & 0.14 & 2224 \\
\hline S Ori J053918.1-025257 & $16.15 \pm 0.08$ & $15.55 \pm 0.10$ & $15.14 \pm 0.13$ & $14.47 \pm 0.17$ & $14.20 \pm 0.18$ & $13.86 \pm 0.20$ & $13.14 \pm 0.20$ & 2224 \\
\hline S Ori 42 & $16.72 \pm 0.13$ & $15.92 \pm 0.13$ & $15.55 \pm 0.21$ & $14.87 \pm 0.21$ & $14.41 \pm 0.20$ & $14.15 \pm 0.23$ & $13.22 \pm 0.20$ & 2224 \\
\hline S Ori 45 & $16.67 \pm 0.11$ & $16.02 \pm 0.13$ & $15.59 \pm 0.21$ & $14.75 \pm 0.37$ & $14.76 \pm 0.23$ & $14.82 \pm 0.33$ & $\gtrsim 14.8$ & 2224 \\
\hline S Ori J053929.4-024636 & $16.96 \pm 0.16$ & $16.52 \pm 0.11$ & $16.03 \pm 0.07$ & $15.17 \pm 0.24$ & $15.18 \pm 0.28$ & $15.38 \pm 0.49$ & $\gtrsim 14.8$ & 1114 \\
\hline S Ori 51 & $17.06 \pm 0.12$ & $16.42 \pm 0.09$ & $16.13 \pm 0.10$ & $15.44 \pm 0.27$ & $15.31 \pm 0.30$ & $15.16 \pm 0.41$ & $\gtrsim 14.8$ & 1114 \\
\hline S Ori 71 & $17.23 \pm 0.07$ & $16.52 \pm 0.11$ & $16.11 \pm 0.05$ & $15.36 \pm 0.26$ & $14.93 \pm 0.25$ & $14.62 \pm 0.30$ & $13.51 \pm 0.24$ & 1114 \\
\hline S Ori J053849.5-024934 & $17.30 \pm 0.08$ & $16.70 \pm 0.05$ & $16.20 \pm 0.07$ & $15.38 \pm 0.26$ & $15.20 \pm 0.29$ & $14.43 \pm 0.26$ & $13.97 \pm 0.30$ & 1114 \\
\hline S Ori 47 & $17.53 \pm 0.24$ & $16.22 \pm 0.17$ & $15.81 \pm 0.08$ & $14.94 \pm 0.21$ & $14.96 \pm 0.26$ & $14.75 \pm 0.30$ & $\gtrsim 14.8$ & 2234 \\
\hline S Ori 50 & $17.50 \pm 0.07$ & $17.12 \pm 0.14$ & $16.18 \pm 0.05$ & $15.60 \pm 0.29$ & \pm 0.36 & $15.07 \pm 0.40$ & & 114 \\
\hline S Ori 53 & 06 & 17. & 16 & $15.94 \pm$ & $15.96 \pm$ & $\gtrsim 15.8$ & & 1114 \\
\hline S Ori J05. & $18.04 \pm 0.08$ & $17.14 \pm 0.32$ & 16.98 & - & 20 & - & & 1102 \\
\hline S Ori J054007.0-023604 & $18.22 \pm 0.07$ & $17.49=$ & 16.8 & $16.07 \pm 0.20$ & 16. & $\gtrsim 15$. & .8 & 1104 \\
\hline S Ori J0s & $18.27 \pm 0.08$ & $17.66 \pm 0.05$ & $17.18 \pm 0.07$ & $16.55 \pm 0.20$ & $16.47 \pm 0.20$ & $\gtrsim 15.8$ & 4.8 & 1114 \\
\hline S Ori J053858.6-025228 & $18.61 \pm 0.08$ & $18.04 \pm 0.06$ & $17.29 \pm 0.07$ & $16.29 \pm 0.20$ & $15.96 \pm 0.20$ & $15.88 \pm 0.60$ & $\gtrsim 14.8$ & 1114 \\
\hline S Ori J053949.5-023130 & $18.89 \pm 0.10$ & $18.18 \pm 0.09$ & $17.42 \pm 0.20$ & $16.57 \pm 0.30$ & $16.62 \pm 0.20$ & $\gtrsim 15.8$ & & 1114 \\
\hline S Ori 60 & $19.05 \pm 0.10$ & $18.00 \pm 0.50$ & $17.30 \pm 0.10$ & $16.52 \pm 0.45$ & $15.52 \pm 0.41$ & $\gtrsim 15.8$ & $\gtrsim 14.8$ & 1134 \\
\hline S Ori 62 & $19.18 \pm 0.10$ & $18.00 \pm 0.50$ & $17.86 \pm 0.10$ & $16.99 \pm 0.34$ & $16.83 \pm 0.48$ & $\gtrsim 15.8$ & $\gtrsim 14.8$ & 1134 \\
\hline S Ori J053844.5-025512 & $19.31 \pm 0.08$ & $18.40 \pm 0.30$ & $17.99 \pm 0.07$ & $16.82 \pm 0.51$ & $16.79 \pm 0.54$ & $\approx 15.8$ & $\gtrsim 14.8$ & 1114 \\
\hline S Ori J054008.5-024551 & $19.43 \pm 0.16$ & - & - & - & - & - & - & 1000 \\
\hline S Ori J053932.4-025220 & $19.54 \pm 0.08$ & $18.70 \pm 0.06$ & $17.86 \pm 0.07$ & $16.12 \pm 0.37$ & $16.23 \pm 0.46$ & $\gtrsim 15.8$ & $\gtrsim 14.8$ & 1114 \\
\hline S Ori J054011.6-025135 & $19.69 \pm 0.09$ & - & - & - & - & - & - & 1000 \\
\hline
\end{tabular}

a Source of the infrared photometry. Three first digits $\left(J H K_{\mathrm{s}}\right): 0$ - not available; 1 - this work; 2 - 2MASS; 3 - Martín et al. (2001). Fourth digit (IRAC passbands): 0 - object in any IRAC channel area; 2 - object in two IRAC channel areas; 4 - object in four IRAC channel areas.

between both $J$-band magnitudes is excellent $(\Delta J=J$ (ISAAC) $J(2 \mathrm{MASS})=-0.03 \pm 0.11 \mathrm{mag})$. However, there are hints for the photometric variability of some targets from the 2MASS epoch $(\mathrm{MJD}=51116.3)$ to the ISAAC epoch $(\mathrm{MJD} \approx 52250)$ which require further studies. Some of them are previously known variables like S Ori 42 (Caballero et al. 2004) and S Ori J053825.4024241 (Caballero et al. 2006a), or are found in this work for the first time (e.g. S Ori J053902.1-023501; $J_{\text {ISAAC }}-J_{2 \text { MASS }}=$ $0.25 \pm 0.12 \mathrm{mag})$.
To complement our $J$-band data and the relatively-shallow $H K_{\mathrm{s}}$-band data from $2 \mathrm{MASS}$, we performed deep ground-based near-infrared imaging of most of the faintest targets in our sample. The follow-up was performed using CFHT-IR at the $3.58-\mathrm{m}$ Canada-France-Hawai' i Telescope $\left(1 \mathrm{k} \times 1 \mathrm{k} ; 0.211 \mathrm{arcsec} \mathrm{pix}^{-1}\right)$, Omega-2000 $(\Omega 2 \mathrm{k})$ at the $3.50-\mathrm{m}$ Calar Alto Telescope $(2 \mathrm{k} \times 2 \mathrm{k}$; $0.450 \operatorname{arcsec}$ pix $^{-1}$ ) and CAIN-II at the 1.52-m Telescopio Carlos Sánchez $\left(256 \times 256 ; 1.00 \operatorname{arcsec}_{\mathrm{pix}}{ }^{-1}\right)$. Table 5 summarises the follow-up campaigns. Each of the CFHT-IR and CAIN-II 
Table 5. Log of the $H K$-band follow-up campaigns.

\begin{tabular}{lcccccccc}
\hline \hline Telescope & Instrument & Filter(s) & Date(s) & $\begin{array}{c}\text { Exp. time } \\
(\mathrm{s})\end{array}$ & $\begin{array}{c}\text { Area } \\
\left(\operatorname{arcmin}^{2}\right)\end{array}$ & $\begin{array}{c}F W H M \\
(\operatorname{arcsec})\end{array}$ & $\begin{array}{c}\text { Compl. } \\
(\mathrm{mag})\end{array}$ & $\begin{array}{c}\text { Limit. } \\
(\mathrm{mag})\end{array}$ \\
\hline CFHT & CFHT-IR & $H, K^{\prime}$ & 2004 Feb. 9,17,22 & 1800 & 85 & $0.7-1.0$ & $20.0-20.5$ & $21.0-21.5$ \\
$3.5 \mathrm{~m} \mathrm{CA}$ & $\Omega 2 \mathrm{k}$ & $H$ & 2003 Oct. 19,22 & $1200-7200$ & $\sim 1100$ & $>1.5$ & 16.8 & 18.1 \\
& & $H, K_{\mathrm{s}}$ & 2005 Jan. 27-Feb. 1 & $5100-11700$ & 480 & $1.3-2.0$ & $17.7-19.5$ & $18.5-20.0$ \\
TCS & CAIN-II & $J, H, K_{\mathrm{s}}$ & 2004 Apr. 19 & $3600-7200$ & $<100$ & $\sim 1.2$ & $18.5,18.0,17.5$ & $19.5,19.0,18.5$ \\
& & $K_{\mathrm{s}}$ & 2006 Oct. 20-22 & $2400-3600$ & $<100$ & $\sim 1.0$ & $\sim 17.5$ & $\sim 18.5$ \\
\hline
\end{tabular}

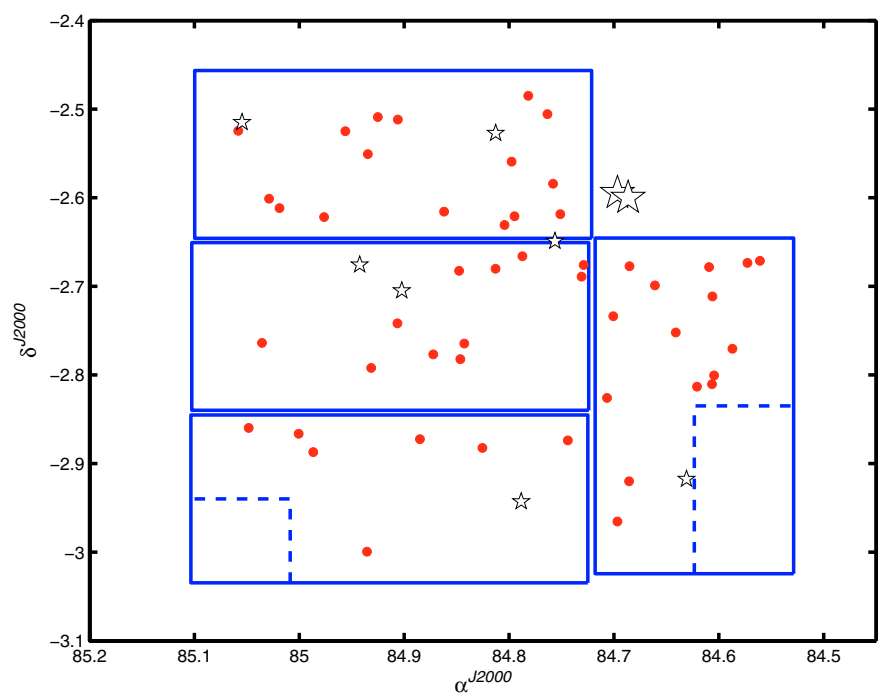

Fig. 3. The $I J$ survey region. The incomplete coverage of the $J$ survey is indicated with dashed lines. Code: filled circles, our cluster members and candidates; big open stars, $\sigma$ Ori $\mathrm{AB}$ and $\mathrm{E}$ (at the centre of the cluster); small open stars, other young stars in the survey area more massive than $1.2 M_{\odot}$ from Caballero (2007). Note the increasing density of objects towards the centre of the cluster.

pointings imaged a single target, whereas several targets were imaged simultaneously in the large field of view of $\Omega 2 \mathrm{k}$. The first run with $\Omega 2 \mathrm{k}$, not as deep as the second, was performed only in the $H$ band, but covering $95 \%$ of the overlapping area between the WFC and ISAAC images. The pointings with CFHT-IR were devoted to the follow-up of four $J$-band sources for which there was no $I$-band counterpart (see Sect. A.2).

For CFHT-IR, we took 30 individual frames of $60 \mathrm{~s}$ exposure time per pointing per filter, using a regular dithering pattern with a suitable shift. A bad pixel mask and a dome flat-field image for each filter were used during the reduction. The $\Omega 2 \mathrm{k}$ observations were similar to those with CFHT-IR, but using shorter exposure times $\left(1.6 \mathrm{~s}-K_{\mathrm{s}}-, 2\right.$ or $\left.3 \mathrm{~s}-H-\right)$ and a random dithering pattern. For CAIN-II, the dithering pattern comprised 10 positions; the individual exposure times were $10 \mathrm{~s}(H)$ and $6 \mathrm{~s}\left(K_{\mathrm{s}}\right)$. Photometric calibration and astrometry procedures for CFHTIR, $\Omega 2 \mathrm{k}$ and CAIN-II were identical to those described for the ISAAC $J$-band images.

The available $J H K_{\mathrm{s}}$ magnitudes of the objects in our sample are provided in Table 4. Apart from the 2MASS catalogue and our ground-based follow-up, we have also taken the $K_{\mathrm{s}}$ magnitude of three candidate cluster members from the literature. The uncertainties in our $H K_{\mathrm{s}}$ magnitudes are, in general, smaller than $0.1 \mathrm{mag}$, and only larger than $0.2 \mathrm{mag}$ in very few cases.

\subsubsection{Blue interlopers and theoretical isochrones}

To summarize, we have collected near-infrared follow-up photometry for 47 selected cluster member candidates: 30 targets with photometry in eight passbands $I J H K_{(\mathrm{s})}[3.5][4.5][5.8][8.0]$; 45 with photometry in at least six of these passbands. We have plotted all the possible colour-magnitude diagrams, in search for "blue interlopers", i.e. possible contaminants that satisfy the $I J$ selection criterion but which display bluer colours than expected for cluster members in other diagrams. In Fig. 4 we show three colour-magnitude diagrams of our sample ( $I$ vs. $I-J, I$ vs. $I-K_{\mathrm{s}}, J$ vs. $J-K_{\mathrm{s}}$ ). Two possible blue-interloper candidates have been identified. From the $J-K_{\mathrm{s}}$ vs. $I-J$ colourcolour diagram in the bottom right panel of Fig. 4, S Ori 51 $\left(I-J=3.16 \pm 0.13 \mathrm{mag} ; J-K_{\mathrm{s}}=0.93 \pm 0.16 \mathrm{mag}\right)$ displays a $J-K_{\mathrm{s}}$ colour that is marginally blue if compared to those of other $\sigma$ Orionis cluster members and field ultracool dwarfs with similar $I-J$ colour (e.g. Dahn et al. 2002). In contrast, its position matches the spectrophotometric cluster sequence in all the colour-magnitude diagrams and its $J$-band spectrum displays low-gravity features, which supports cluster membership (McGovern et al. 2004). The second one, S Ori J053922.2$024552\left(I=17.04 \pm 0.03 \mathrm{mag} ; I-K_{\mathrm{s}}=2.63 \pm 0.09 \mathrm{mag}\right)$, without spectroscopic information, has an $I-K_{\mathrm{s}}$ colour that is slightly bluer than the confirmed cluster members of the same magnitude in our survey. It has, however, similar colours and magnitudes to SE 70, a $\sigma$ Orionis brown dwarf with lithium in absorption, X-ray flares, and low-gravity features (Burningham et al. 2005a; Caballero et al. 2006b). We keep both S Ori 51 and S Ori J053922.2-024552 in the sample. We cannot determine whether the two faint objects with only $I$ - and $J$-band photometry available, S Ori J054008.5-024551 (previously classified as high-probability cluster member in the $i^{\prime} z^{\prime}$-band search with $J$-band follow-up by González-García et al. 2006) and S Ori J054011.6-025135, are blue contaminants or not.

We have compared our selection criterion and the cluster sequence from our data with model evolutionary tracks at very young ages. For this purpose we used the isochrones of the Lyon group (Baraffe et al. 1998 - NextGen98; Chabrier et al. 2000 Dusty00; Baraffe et al. 2003 - Cond03) at different ages and at the most probable heliocentric distance of $\sigma$ Orionis. The Dusty00 isochrone at $3 \mathrm{Ma}$ acts as a good separator at $I<20 \mathrm{mag}$ in the $I$ vs. $I-K_{\mathrm{s}}$ diagram. To sum up, no blue outliers have been identified using either the empirical cluster sequences or the model isochrones (Fig. 4).

\section{Results and discussion}

\subsection{Individual masses}

Modelling ultracool atmospheres is a complicated issue, because it strongly depends on the chemical composition, the dust 

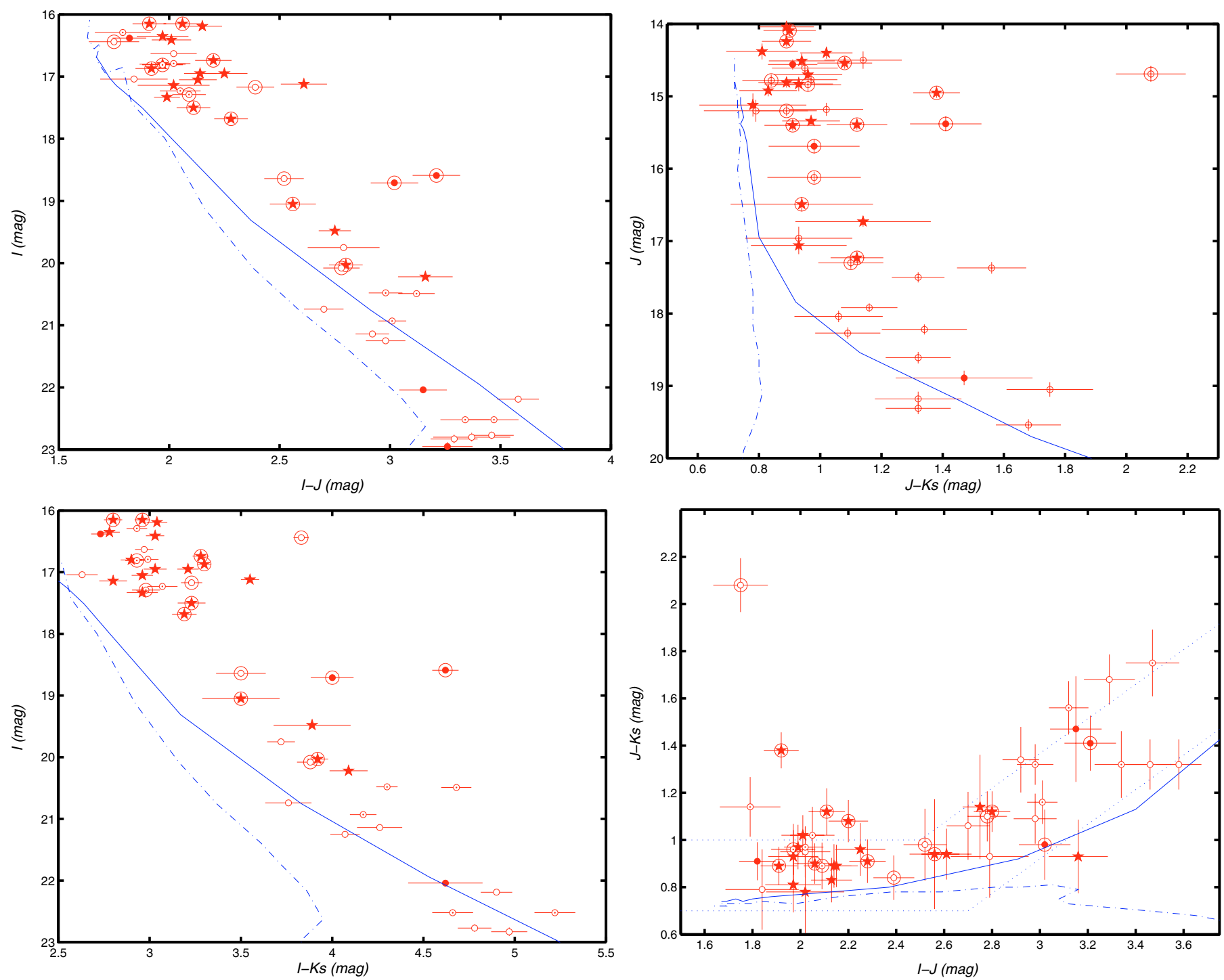

Fig. 4. $I$ vs. $I-J$ (top left), $I$ vs. $I-K_{\mathrm{s}}$ (bottom left), and $J$ vs. $J-K_{\mathrm{s}}$ (top right) colour-magnitude diagrams, and $J-K_{\mathrm{s}}$ vs. $I-J$ colour-colour diagram (bottom right). $I$ is from WFC03, $J$ from ISAAC and $H K_{\mathrm{s}}$ from the ground-based follow-up data. The symbols are as in Fig. 2 . Objects with discs from IRAC excess flux are marked with big circles. Solid and dash-dotted lines are the Dusty00 and Cond03 3 Ma-old isochrones at $360 \mathrm{pc}$ of the Lyon group. Dotted lines in the colour-colour diagram mark the approximate boundaries of the sequence of field ultracool dwarfs (Dahn et al. 2002). The very red object with $I-J=1.75 \pm 0.11 \mathrm{mag}$ and $J-K_{\mathrm{s}}=2.08 \pm 0.11 \mathrm{mag}$ is S Ori J053902.1-023501, whose spectral energy distribution (Fig. 6) shows evidence for a circumstellar disc (Sect. 4.2).

content, the amount of condensation and the size distribution of any dust grains (Tsuji et al. 1996; Allard et al. 2001; Baraffe et al. 2002). To determine the masses, $M$, of our objects, we will rely on the estimated luminosity, $L$, and mass-luminosity relations predicted by theoretical models of their interior, rather than on derived magnitudes in each passband. In particular, we use the $M-\log \frac{L}{L_{\odot}}$ relations of the Lyon group and the basic properties of $\sigma$ Orionis provided in Section 1 (age, distance, metallicity). The mass-luminosity relations given by the NextGen98, Dusty00, and Cond03 models are identical.

The luminosity for each target was computed from the bolometric magnitude, $M_{\mathrm{bol}}=M_{\mathrm{bol}, \odot}-2.5 \log \frac{L}{L_{\odot}}\left(\right.$ where $M_{\mathrm{bol}, \odot}=$ $4.74 \mathrm{mag}$; Cox 2000). The bolometric magnitude is:

$M_{\mathrm{bol}}=M_{J}^{0}+B C_{J}$

where $M_{J}^{0}=M_{J}\left(J, d, A_{J}=0\right)$ is the absolute $J$-band magnitude assuming no extinction and $B C_{J}$ is the bolometric correction in the $J$ band and $d$ is the heliocentric distance. Caballero (2006) compiled from the literature $I$ - and $J$-band photometry, parallaxes and luminosity determinations for all ultracool dwarfs known at the time. From these he computed a bolometric correction in $J$ as a function of the $I-J$ colour:

$B C_{J}(I-J)=a_{3}(I-J)^{3}+a_{2}(I-J)^{2}+a_{1}(I-J)+a_{0}$

with $a_{3}=+0.091 \mathrm{mag}^{-2}, a_{2}=-0.875 \mathrm{mag}^{-1}, a_{1}=+2.486$, $a_{0}=-0.140 \mathrm{mag}$. The standard deviation of the mean of the fit was $\sigma\left(B C_{J}\right)=0.15 \mathrm{mag}$. The bolometric correction $B C_{J}$ is not strongly dependent on the $I-J$ colour. It varies between $1.75 \pm 0.15$ and $2.06 \pm 0.15 \mathrm{mag}$ in the $1.5 \mathrm{mag}<I-J<4.0 \mathrm{mag}$ interval. The input data for this relation are the same as for other relations between $B C_{K}, M_{J}, I-J, J-K, K-L^{\prime}$ and spectral type of ultracool dwarfs found in the literature (Dahn et al. 2002; Golimowski et al. 2004; Vrba et al. 2004). Recent measurements of the bolometric magnitudes of ultracool dwarfs 
using IRS/Spitzer spectra support previous $M_{\mathrm{bol}}$ determinations (Cushing et al. 2006). By using this $B C_{J}(I-J)$ relation, we assume that the spectral energy distribution in field ultracool dwarfs and very young cluster members is similar. The derivation of the $M_{\mathrm{bol}}$ from the $J$-band magnitude minimises possible contributions to the total error by flux excesses at longer wavelengths or photometric variability at bluer wavelengths (as in the case of T Tauri stars and substellar analogs).

The adopted distance modulus for the $\sigma$ Orionis cluster is $m-M=7.78 \pm 0.42 \mathrm{mag}$ (Brown et al. 1994). The uncertainty of $20 \%$ in the determination of the cluster distance $\left(d=360_{-60}^{+70} \mathrm{pc}\right)$, together with the uncertainty in the age $(3 \pm 2 \mathrm{Ma})$, are the most important contributors to the final error in the mass of each target (the uncertainties in the distance may be even larger; Caballero 2007). Errors coming from the photometric uncertainty or the use of the $B C_{J}(I-J)$ relation are comparatively smaller. Theoretical models are also a source of systematic uncertainty at very young ages (Baraffe et al. 2002). Although there is reasonable criticism on the validity of evolutionary tracks at very low masses and very young ages (less than $10 \mathrm{Ma}$ ), the observational determination of the mass-age-luminosity triplet at different ages in the substellar domain is in agreement with theoretical predictions (Bouy et al. 2004; Zapatero Osorio et al. 2004; Stassun et al. 2006).

We give in the last columns of Table 3 the pair $M-\log \frac{L}{L_{\odot}}$ for the 49 cluster members and member candidates. The corresponding errors in mass and luminosity account for the propagation of uncertainties from the $J$-band magnitude as well as the age and distance the of $\sigma$ Orionis cluster. The theoretical effective temperatures derived from the $\log \frac{L}{L_{\odot}}-T_{\text {eff }}$ relation (between $3030 \pm 120$ and $1740 \pm 70 \mathrm{~K}$ ) roughly match the expected effective temperatures of objects with spectral type determination. Five objects have most probable masses larger than the hydrogen burning mass limit and they are thus very low-mass stars. Among the other 44 substellar objects, 11 are planetarymass object candidates and 33 are brown dwarf candidates. The masses of some of our objects have been previously determined (Zapatero Osorio et al. 2000; Béjar et al. 2001; Caballero et al. 2006a; González-García et al. 2006). These differ by less than $10 \%$ from our values.

\subsection{Infrared excesses and discs}

\subsubsection{Detection of infrared excesses}

Brown dwarfs in very young regions have, like T Tauri stars, circum(sub)stellar discs (Wilking et al. 1999; Natta \& Testi 2001; Fernández \& Comerón 2001; Jayawardhana et al. 2003; Furlan et al. 2005). With our follow-up, we have been able to measure flux excesses redwards of $2 \mu \mathrm{m}$ in some of our targets, which are very probably associated with the presence of circum(sub)stellar discs. Objects with discs are extremely young ( $\$ 10 \mathrm{Ma})$, which confirms membership in $\sigma$ Orionis $^{1}$. We have investigated different ISAAC/2MASS/IRAC colour-colour magnitude diagrams to search for infrared excesses in our cluster members and candidates. The [3.6] - [8.0] vs. $J-K_{\mathrm{s}}$ diagram (Fig. 5) illustrates the difference in the colour between objects without discs $(0.0 \mathrm{mag} \lesssim[3.6]-[8.0] \lesssim 0.5 \mathrm{mag})$ and those that very probably harbour discs ([3.6]-[8.0] $\gtrsim 1.0 \mathrm{mag})$. The latter display flux excesses in the [5.8] and [8.0] bands with respect to the [3.6] band

1 Jeffries et al. (2006) and Caballero (2007) have discussed the spatial overlapping of different young populations of the Ori OB $1 \mathrm{~b}$ Association in the direction of $\sigma$ Orionis.

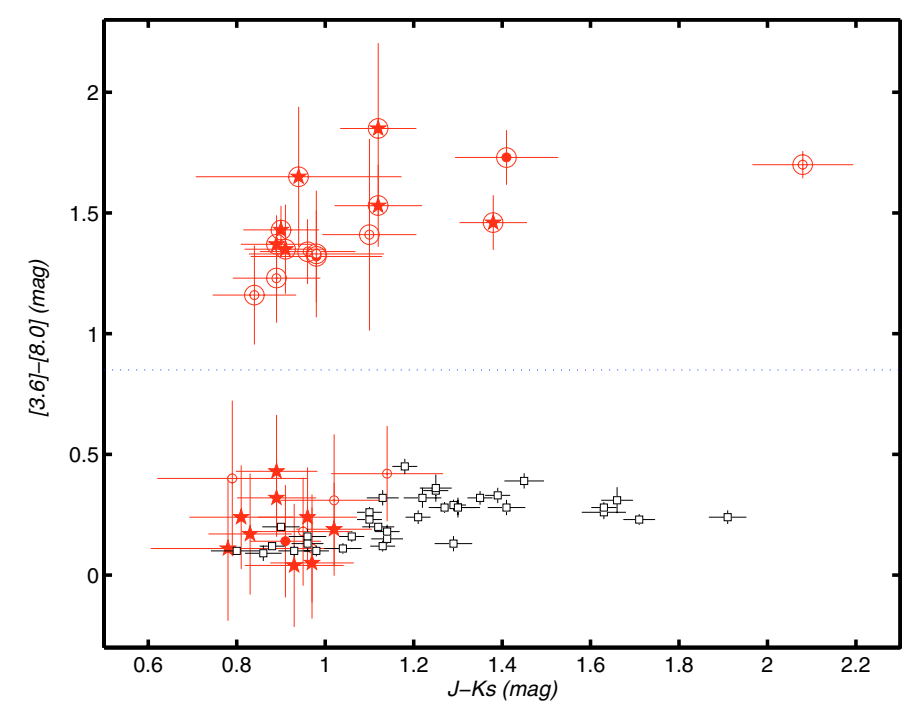

Fig. 5. [3.6] - [8.0] vs. $J-K_{\mathrm{s}}$ colour-colour diagram. The symbols are as in Figs. 2 and 4. The dotted line marks the boundary between objects with and without discs. Field ultracool dwarfs with spectral types in the range M3.0-L5.0 from Patten et al. (2006) are shown with open (black) squares.

that are not found in any of the 60 or so ultracool field dwarfs earlier than T3V studied with IRAC/Spitzer by Patten et al. (2006).

Of the 30 cluster members and candidates with IRAC detection at [3.6] and [8.0], 15 have colours [3.6] - [8.0] > $0.90 \mathrm{mag}$, which we classify as objects with discs. Given the extremely red $K_{\mathrm{s}}-[8.0]$ colour of the confirmed cluster member S Ori J053847.2-025756, of $2.04 \pm 0.09 \mathrm{mag}$, we will also classify it as an object with disc, although it has only IRAC photometry at [4.5] and [8.0]. The 16 probable objects with discs are marked with a "D" in Table 3 . Our criterion is consistent with other disc selections in the literature based on IRAC data. In a very recent paper, Hernández et al. (2007) study the spectral energy distributions (SEDs) of most of our targets brighter than $J=16.0 \mathrm{mag}$. They classify the objects according to their $K_{\mathrm{s}}-$ [24] colours as brown dwarfs without excess, with optically-thick discs, and with "evolved discs". We confirm all their disc detections for brown dwarfs brighter than $J$ $=16.0 \mathrm{mag}$, and find a new one surrounding S Ori J053847.2025756, which is not in their investigated area. They also find a transition disc around S Ori J053954.3-023719 (evident only at the Spitzer/MIPS $24 \mu \mathrm{m}$ band) - marked with “(D)" in Table 3.

\subsubsection{Properties of the disc harbours}

In Table 2 there are six cluster members that display strong and/or broad $\mathrm{H} \alpha$ emission. Three satisfy the accretion criterion of Barrado y Navascués \& Martín (2003) (S Ori J053825.4024241, S Ori 42 and S Ori 71), while for the remaining three there are only qualitative estimates of the broadening of the line. The width of the $\mathrm{H} \alpha$ line at $10 \%$ of the peak has been only measured for S Ori 25 and S Ori 45, so the White \& Basri (2003) accretion criterion cannot be applied here. S Ori 71 is one of the strongest $\mathrm{H} \alpha$ emitters close to the deuterium-burning mass limit. S Ori J053825.4-024241 displays forbidden emission lines and is a very red object $\left(J-K_{\mathrm{s}}=1.38 \pm 0.08 \mathrm{mag}\right.$, [3.6] $-[8.0]=1.46 \pm 0.11 \mathrm{mag})$. There are hints of optical photometric variability in all six cases (Caballero et al. 2004). S Ori J053825.4-024241 and S Ori 42 are also variable in the 


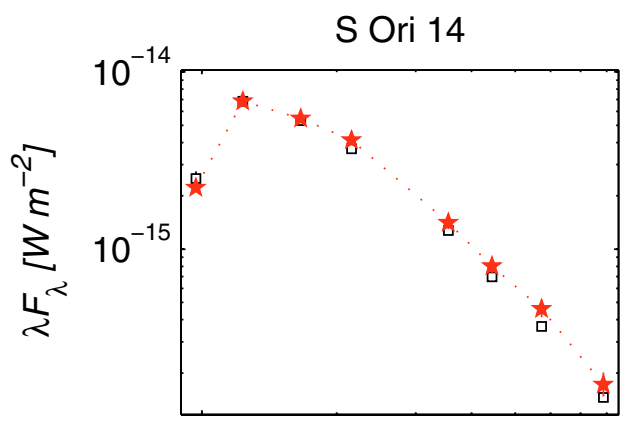

S Ori J053825.4-024241

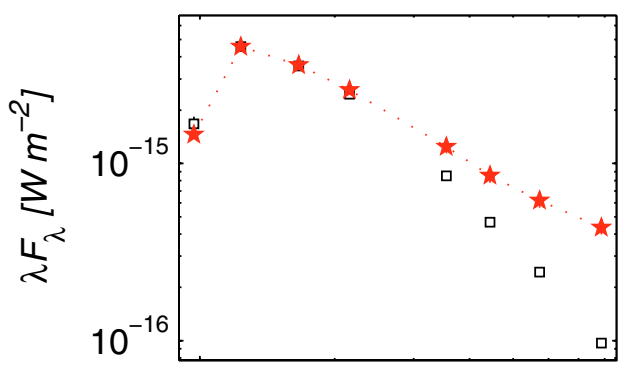

S Ori 42

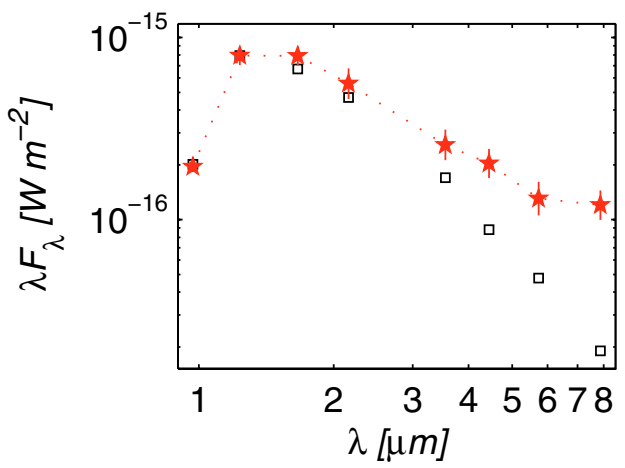

S Ori J053838.6-024157

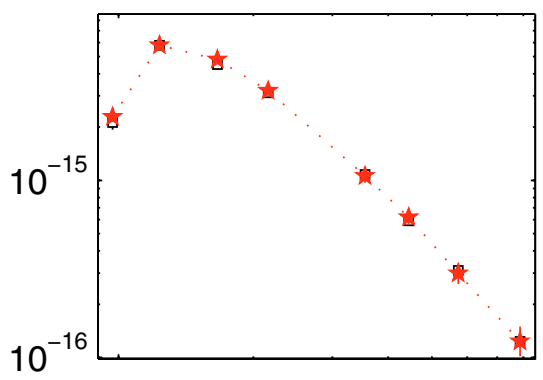

S Ori 32

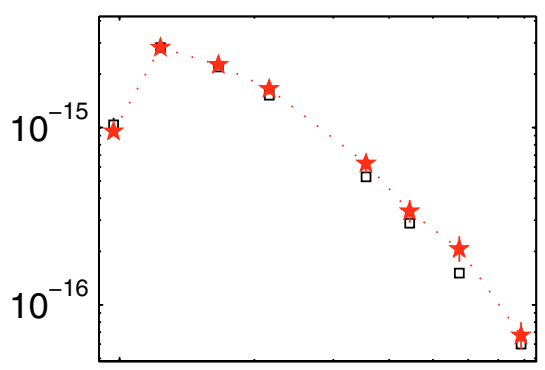

S Ori 71



S Ori J053902.1-023501

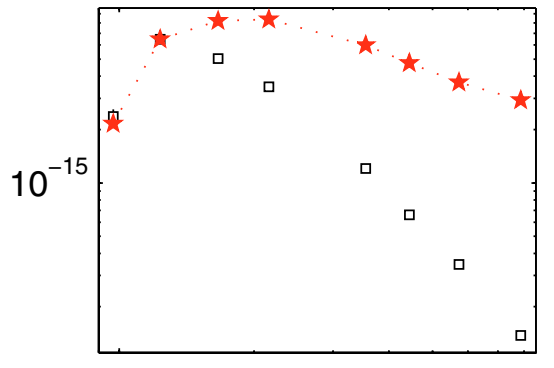

S Ori 36

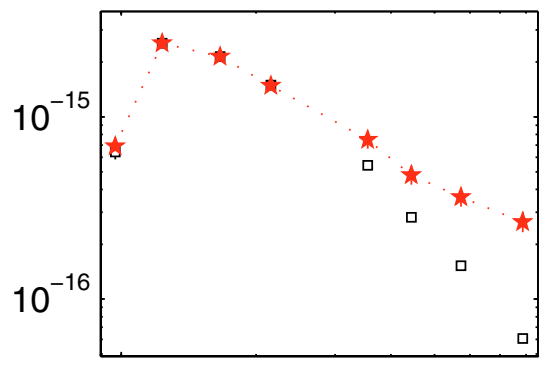

S Ori J053849.5-024934



Fig. 6. Spectral energy distributions from the $I$ band to the [8.0] band of nine representative substellar objects in $\sigma$ Orionis (filled stars). The open squares indicate the SEDs of ultracool field dwarfs of similar spectral type, normalised at the $J$ band.

$J$ band, as discussed in Sect. 3.3.2. All these objects, except S Ori J054014.0-023127, which is not in the IRAC survey area, have flux excesses at [5.8] and [8.0] (and at [24]; Hernández et al. 2007). S Ori J054014.0-023127 is a photometric variable and has an $I-J$ colour of $2.61 \pm 0.10 \mathrm{mag}$ (this is red for its $I$ band magnitude) and a marginally broad $\mathrm{H} \alpha$ emission (Kenyon et al. 2005). There are no $\mathrm{H} \alpha$ measurements for the remaining cluster members with discs.

The SEDs of nine representative confirmed brown dwarfs, six with discs, are shown in Fig. 6. The flux excesses at long wavelengths with respect to the expected fluxes from ultracool dwarfs of roughly the same $I-J$ colours are evident in the six objects with discs. The comparison field dwarfs, with infrared data from Patten et al. (2006), are GJ 1002 (M5.5), DX Cnc (GJ 1111; M6.5), vB 8 (V1054 Oph E, M7.0), and 2MASS J12043036+3212595 (M9.0).

\subsubsection{Frequency of brown dwarfs with discs}

According to the masses listed in Table 3, there are 2 stars and 14 brown dwarfs with discs (as indicated by an excess emission at $8.0 \mu \mathrm{m}$ ). Taking into account the completeness magnitude at
[8.0] and that objects with discs have colours [3.6] $-[8.0]>$ $0.90 \mathrm{mag}$, our 3.6-8.0 $\mu \mathrm{m}$ IRAC photometry is complete down to $\sim 0.015 M_{\odot}$, i.e. over most of the brown dwarf mass interval. We estimate the frequency of brown dwarfs with discs in $\sigma$ Orionis as $47 \pm 9 \%$ (14 brown dwarfs with infrared excess among 30 cluster members and candidates with masses $0.072 M_{\odot}>\mathrm{M}>$ $0.015 M_{\odot}$ and detection in the four IRAC channels; binomial error). If we consider S Ori J053954.3-023719 (with a transition disc according to Hernández et al. 2007) and some possible foreground ultracool contaminants, the disc frequency could exceed $50 \%$.

The value of $47 \pm 9 \%$ is comparable to or slightly larger than other determinations of the frequency of discs surrounding stars and brown dwarfs in the cluster. For example, the spectroscopically derived ratio of classical $\mathrm{T}$ Tauri stars to weak-line $\mathrm{T}$ Tauri star in $\sigma$ Orionis is $30-40 \%$ (Zapatero Osorio et al. 2002a). From infrared colours (observed flux excesses in the $L^{\prime}$-band and/or in the IRAC+MIPS/Spitzer passbands), Oliveira et al. (2006) and Hernández et al. (2007) found cluster disc frequencies of $27-39 \%$ in the mass range $1.0-0.04 M_{\odot}$. Hernández et al. (2007) did not observe any significant decrease in the disc frequency towards the brown dwarf domain. Our result, which is 
Table 6. Estimate of ultracool field dwarf contaminants.

\begin{tabular}{lccccccccccc}
\hline \hline $\begin{array}{l}\Delta I \\
(\mathrm{mag})\end{array}$ & M3-5 & M5-7 & M7-9 & M9-L1 & L1-3 & L3-5 & L5-7 & L7-T0 & T0-2 & T2-8 & $\begin{array}{c}\text { All } \\
\text { Sp. T. }\end{array}$ \\
\hline $16.1-17.1$ & 0 & 0.45 & 0.00 & 0 & 0 & 0 & 0 & 0 & 0 & 0 & 0.45 \\
$17.2-18.2$ & 0 & 0.25 & 0.03 & 0 & 0 & 0 & 0 & 0 & 0 & 0 & 0.28 \\
$18.3-19.3$ & 0 & 0 & 0.14 & 0.01 & 0.00 & 0 & 0 & 0 & 0 & 0 & 0.15 \\
$19.4-20.4$ & 0 & 0 & 0.60 & 0.04 & 0.01 & 0.00 & 0 & 0 & 0 & 0 & 0.65 \\
$20.5-21.6$ & 0 & 0 & 0.74 & 0.17 & 0.05 & 0.01 & 0.00 & 0 & 0 & 0 & 0.97 \\
$21.7-22.7$ & 0 & 0 & 0.43 & 0.68 & 0.23 & 0.05 & 0.01 & 0.00 & 0 & 0 & 1.40 \\
$22.8-23.8$ & 0 & 0 & 0 & 0.86 & 0.78 & 0.22 & 0.06 & 0.01 & 0.01 & 0 & 1.94 \\
$23.9-24.9^{a}$ & 0 & 0 & 0 & 0 & 0.09 & 0.28 & 0.13 & 0.02 & 0.02 & 0.01 & 0.55 \\
\hline All intervals $^{a}$ & 0 & 0.70 & 1.94 & 1.76 & 1.16 & 0.56 & 0.20 & 0.03 & 0.03 & 0.01 & 6.39 \\
\hline
\end{tabular}

Assuming an incompleteness factor of 0.5 in the magnitude interval $23.9-24.9$ mag.

among the first disc frequency determinations in the mass interval $0.075-0.015 M_{\odot}$ (see also the survey in Taurus by Luhman et al. 2006), combined with the cluster stellar data from the literature, suggests little dependence of the disc frequency with mass, from solar masses down to $0.015 M_{\odot}$. This supports theoretical scenarios where brown dwarfs form as a result of an extension of the low-mass star formation process (see references in Jameson 2005).

\subsection{Contamination by fore- and background sources}

Taking together the 20 confirmed cluster members in Table 2 and the 18 cluster member candidates with discs in Table 3, from which eight are also previously confirmed cluster members, then 30 objects of our 49 candidate cluster members exhibit signatures of extreme youth and, therefore, are bona fide cluster members. Out of the remaining 19 candidate cluster members without known youth features, nine have low-resolution spectroscopy and ten have no spectroscopic information or indications of discs from the IRAC photometry. For two very faint objects there is no near-infrared follow-up at all.

There could be contamination by red giants, galaxies and field dwarfs among our targets awaiting membership confirmation. All our cluster member candidates are far from the Galactic plane $(b=-17.3 \mathrm{deg})$ and have point-like PSF, so contamination by red giants or galaxies is unlikely. Besides, the 47 objects with near-infrared follow-up display colours that match the dwarf sequence in a colour-magnitude diagram (e.g. $J-K_{\mathrm{s}}$ vs. $I-J$ in Fig. 4). Therefore, we estimate the contamination fraction in our $I J$ survey only from background and foreground non-member field dwarfs of very late spectral types (intermediate- and late$\mathrm{M}, \mathrm{L}$, and T). This we base on up-to-date models and data from the literature: $(i)$ an exponential model for the Galactic thin disc (Phleps et al. 2005; Ryan et al. 2005; Karaali 2006); (ii) the length and height scales for late-type dwarfs in the Galaxy (Chen et al. 2001); (iii) the spatial densities, absolute magnitudes and colours of ultracool dwarfs for each spectral type (Kirkpatrick et al. 1994; Dahn et al. 2002; Cruz et al. 2003; Vrba et al. 2004; Nakajima 2005).

Table 6 shows the resulting possible contaminants listed in approximately 1 mag-wide bins. Since the last magnitude interval (23.9-24.9 mag) is fainter than our completeness limit, we have used an incompleteness factor of 0.5 , i.e. only $\sim 50 \%$ of the sources in this interval are detected in the WFC data. The total number of possible field ultracool-dwarf contaminants in our survey is $\sim 6$. Most of them are M7-L4V dwarfs in the magnitude interval $I=20-24$ mag. The contribution to contamination

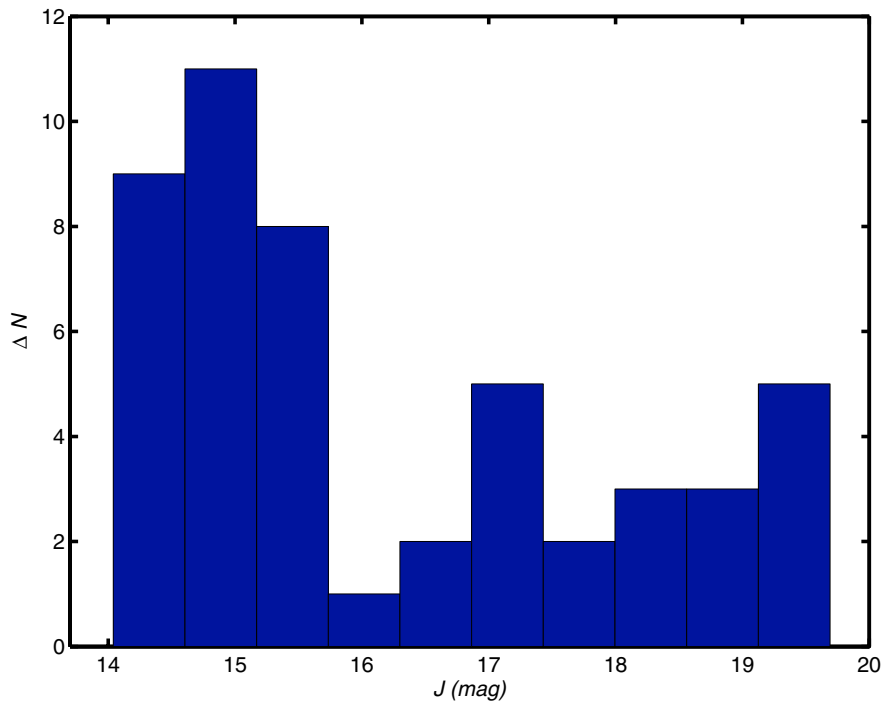

Fig. 7. Luminosity function in the $J$-band. Note the scarcity of objects at $J \sim 16.0$ mag.

by dwarfs later than L5 is very small. The figures reasonably match other determinations of the number of contaminants along the line of sight to $\sigma$ Orionis (e.g. Béjar et al. 1999; Zapatero Osorio et al. 2002c; González-García et al. 2006). Our contamination calculations predict that $\sim 4$ of the $\sim 6$ foreground ultracool dwarfs are in the expected magnitude interval of the PMOs in the cluster.

\subsection{The mass function in the substellar domain}

In Fig. 7, we show the luminosity function in $J$-band. There is an abrupt discontinuity in the distribution of magnitudes of brown dwarfs at $J \sim 16.0 \mathrm{mag}, I-J \sim 2.5 \mathrm{mag}$, which translates into a break in the mass distribution at $\sim 0.03 M_{\odot}$ for an age of $3 \mathrm{Ma}$. In fact, there are only two cluster members with $J$-band magnitude between 15.7 and $16.7 \mathrm{mag}$.

The number of objects within each mass interval and the mass spectrum $(\Delta N / \Delta M)$ from our data with and without contamination correction are shown in Fig. 8. We mark three mass intervals in the substellar regime which we use to classify our objects in high-mass brown dwarfs $\left(0.073-0.032 M_{\odot}\right)$, lowmass brown dwarfs $\left(0.032-0.013 M_{\odot}\right)$ and planetary-mass objects $\left(0.013-0.006 M_{\odot}\right)$. The lowest mass interval is complete down to $0.006 M_{\odot}$ for the most probable age of the cluster. These 


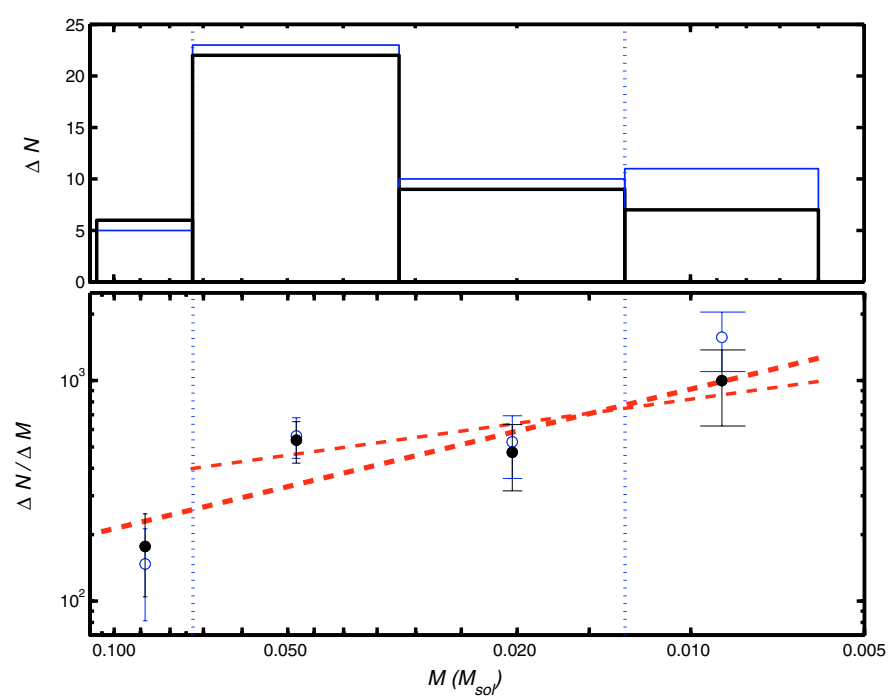

Fig. 8. Number of objects within each mass interval (top panel) and mass spectrum (bottom panel) from 0.110 to $0.006 M_{\odot}$. Dotted vertical lines denote the hydrogen (left) and deuterium (right) burning mass limits. The values not corrected for contamination are shown with thin lines (top panel) and open circles (bottom panel), while the values corrected for contamination are shown with thick lines (top panel) and filled circles (bottom panel). The fits of the mass spectrum in the three substellar bins (thin dashed line) and the four bins (thick dashed line) are also shown.

three mass intervals are contaminated by 1,1 , and 4 field ultracool dwarfs respectively (see Sect. 4.3). In contrast, the stellar mass domain $\left(0.11-0.073 M_{\odot}\right)$ has no appreciable contamination by $\mathrm{M}$ and early-L dwarfs. However, as mentioned in Sect. 2.3, the survey is not complete at the stellar mass end due to saturation effects. The survey area and the magnitude interval corresponding to very-low-mass stars have been intensively investigated by other authors (Béjar et al. 1999, 2001; Scholz \& Eislöffel 2004; Sherry et al. 2004), and they did not find any additional targets fainter than $J=14.2 \mathrm{mag}$. We estimate that up to one cluster star between $0.11 M_{\odot}$ and the hydrogen burning limit may have been missed.

The best fit for the four decontaminated intervals and for the most probable age and distance gives an $\alpha$ power index of $\sim 0.65\left(\Delta N / \Delta M \approx a M^{-\alpha}\right)$. Varying the widths of the mass bins, the $\alpha$ index changes between 0.4 and 0.8 . We will assume $\alpha=+0.6 \pm 0.2$ as the slope of the mass spectrum in the interval $0.11-0.006 M_{\odot}$. This value is similar to other determinations of the slope in the low-mass star and substellar domain in $\sigma$ Orionis $\left(\alpha=+0.8 \pm 0.4\right.$, Béjar et al. 2001; $\alpha=+0.6_{-0.1}^{+0.5}$, González-García et al. 2006). An extrapolation of the mass spectrum with index $\alpha=+0.6 \pm 0.2$ predicts $3-4$ objects with masses $0.005-0.003 M_{\odot}$ in the area of our survey. Taking into account only the three substellar mass intervals, the slope decreases down to $\alpha=+0.4 \pm 0.2$. It is equal within the error bars to the $\alpha$ value proposed by Kroupa (2001) between 0.080 and $0.010 M_{\odot}$ $(\alpha=+0.3 \pm 0.7)$.

Photometric variability, mass-segregation or unresolved binarity corrections have not been applied in any mass interval for the following reasons:

- Our $I$ and $J$ images were not taken simultaneously, so photometric variability can potentially affect the selection procedure in the sense that variable objects may be missed because they lie to the blue of our selection criterion. Non-simultaneous photometric surveys in young regions in general, and in our survey in particular, may yield incomplete lists of candidates. However, as noted previously, our $J$-band images have been combined with $I$-band data taken on two different occasions, and the resulting sample of $\sigma$ Orionis candidates is not changed. Furthermore, in Caballero et al. (2004) and in Scholz \& Eislöffel (2004) it was found that the amplitude of the photometric variations of $\sigma$ Orionis brown dwarfs is typically less than 0.15 mag in the I-band (only one source was observed with larger amplitude). Our selection criterion can account for such small variability, but does not consider larger amplitude variations, such as those possibly observed for S Ori J053948.1-022914 (see Sect. A.1). We estimate the incompleteness of our sample at about $2 \%$ due to photometric variability effects. This rather small fraction does not seem to have a relevant impact on the study of the cluster substellar mass funtion (see Burningham et al. (2005b) for a discussion on apparent age spreads in OB association colour-magnitude diagrams and variability).

- No significant mass-dependent difference between the spatial distributions of cluster members below 3.7 $M_{\odot}$ has been found in $\sigma$ Orionis (Béjar et al. 2004; Caballero, in prep.). Only the most massive stars $\left(M>3.7 M_{\odot}\right)$ are preferentially found towards the centre of the cluster. In particular, the four most massive stars in $\sigma$ Orionis are in the multiple stellar system that gives the name to the cluster (Caballero 2007). Hillenbrand \& Hartmann (1998) found no evindence for mass segregation below $\sim 1-2 M_{\odot}$ in the Orion Nebula Cluster (age $<1 \mathrm{Ma}$ ). Given the extreme youth of the starforming regions in Orion, there may not have been enough time for mass segregation as is seen in older open clusters, such as the Pleiades or the Hyades (Pinfield et al. 1998; Perryman et al. 1998), or in very old globular clusters.

- Little is known about binarity in $\sigma$ Orionis, especially at substellar masses. Only a few works have found multiple systems in the cluster with low-mass components (Lee et al. 1994 and Kenyon et al. 2005: spectroscopic binaries; Caballero 2005 and Caballero et al. 2006b: wide binaries and companions). Unresolved binaries of mass ratio close to 1 are $\sim 0.7$ mag brighter than single objects, which leads to higher derived masses. Some currently considered single low-mass stars could really be tight brown dwarf pairs. However, more radial-velocity investigations and high spatial resolution imaging are needed to quantify the binary frequency in $\sigma$ Orionis (see Köhler et al. 2006 for a study of binary frequency in the Orion Nebula Cluster).

Our list of cluster members may also be incomplete because the regions around very bright stars show extensive glare which occupies several times the typical FWHM of the I-band images. However, the total lost area because of this effect is less than $2 \%$ (see previous sections). Given the spatial density of freefloating $\sigma$ Orionis members in the investigated magnitude intervals (49 in $790 \mathrm{arcmin}^{2}$, i.e. 0.062 objects $\operatorname{arcmin}^{-2}$ ), we estimate the number of missed cluster members to be less than one.

Our mass spectrum extends to mass regimes not well sampled in the literature, limiting the opportunity for comparison. Yet our derived mass function for $\sigma$ Orionis cluster is consistent with that derived by Lucas \& Roche (2000); Najita et al. (2000); Muench et al. (2002) and Slesnick et al. (2004) in other regions. This is not a strong statement, however, given the low number of objects per mass bin.

The sharp drop observed at $J \sim 16$ mag $\left(M \sim 0.03 M_{\odot}\right)$ in the $\sigma$ Orionis luminosity function of Fig. 7 is not explained by 
any theoretical evolutionary model currently available in the literature (Burrows et al. 1997; Baraffe et al. 1998, 2003; Chabrier et al. 2000). Similar drops or breaks at comparable magnitudes are also found in the near-infrared luminosity-functions of some recent deep searches conducted in various young clusters and star-forming regions (Muench et al. 2003 in IC 348; Pinfield et al. 2003 in the Pleiades and the Praesepe; Lucas et al. 2005 in the Orion Nebula Cluster; Bihain et al. 2006 in the Pleiades). Muench et al. (2002) discussed an apparent break from a single power-law decline of the Trapezium brown dwarf mass function around $0.02-0.03 M_{\odot}(K \approx 15.5 \mathrm{mag})$. Their mass function shows a second peak near the deuterium-burning limit, followed by a rapid decline to lower masses. Both the Trapezium and $\sigma$ Orionis clusters have similar characteristics (age, distance, metallicity, environment). It is likely that Muench et al. (2002)'s break and ours share a common origin. Dobbie et al. (2002) also found that the luminosity functions of star-forming regions, open clusters and the field show a drop between spectral types M7 and M8, which corresponds to colours $I-J \sim 2.2-2.5 \mathrm{mag}$. As is apparent from Fig. 2, the sharp drop of the $\sigma$ Orionis luminosity function also lies in this colour interval. Dobbie et al. (2002) speculated that this is caused by the beginning of dust formation in cool, "neutral" atmospheres. To date, no satisfactory explanation exists to account for this feature.

Finally, we have investigated the effects of the uncertainties in the cluster age and distance on the mass spectrum. In particular, the slope of the mass spectrum is highly sensitive to age variations, which prevents us from deriving the error in the $\alpha$ index with a precision better than 0.2 . The break at $M \sim 0.03 M_{\odot}$ did not affect our first $\alpha$ determination, since we used $0.032 M_{\odot}$ as the boundary between high- and low-mass brown dwarfs in the mass spectrum. However, the break is displaced to the middle of the intervals in the mass spectrum for the extreme cases of the cluster being younger and closer ( $1 \mathrm{Ma}$ and $300 \mathrm{pc}$ ) and older and further away ( $5 \mathrm{Ma}$ and $430 \mathrm{pc}$ ). For these cases, one of the two brown dwarf intervals is almost empty, and the linear fit becomes meaningless. Using more mass intervals does not solve the problem. A diagram with the normalised cumulative number of objects as a function of mass, like the one shown in Fig. 9, is free of the subjectiveness in the choice of mass intervals in the mass spectrum and appears useful to compare the distribution of masses of stars and substellar objects in different young clusters. There is an apparent change of slope in the cumulative distribution of Fig. 9 at masses between 0.025 and $0.040 M_{\odot}$. This is likely related to the sharp drop observed in the cluster luminosity function.

Our main goal was to investigate whether there is any feature in the mass function that could be linked to the opacity masslimit for objects forming via fragmentation in molecular clouds. At lower masses, our luminosity function is rather smooth with no evidence for a theoretically predicted opacity mass-limit. We derived a most probable mass of $0.006 M_{\odot}$ for our least massive cluster member candidate. Besides, there are seven IPMO candidates with most probable masses in the interval $0.008-0.006 M_{\odot}$. This is a relatively large number, even accounting for possible contamination. If there were an opacity limit just below the deuterium-burning mass limit, we would expect a lower number of cluster member candidates in that mass interval, which would lead to a steep drop in the last bin of the mass spectrum in Fig. 8. Thus, if these objects form via fragmentation, any possible mass cut-off of the mass function lies below $0.006 M_{\odot}$ $\left(6 M_{\text {Jup }}\right)$. The smooth continuity of the substellar mass spectrum towards masses below the deuterium-burning limit may indicate that the formation of IPMOs with $M>0.006 M_{\odot}$ is merely an



Fig. 9. Normalised cumulative number of objects $\left(\sum_{i} i / N\right)$ vs. mass. Filled circles indicate the most probable masses for $3 \mathrm{Ma}$ and $360 \mathrm{pc}$. The error bars indicate the masses for the extreme cases of a younger closer cluster (1 Ma and $300 \mathrm{pc}$ ) and of an older farther cluster (5 Ma and $430 \mathrm{pc}$ ). Dotted vertical lines denote the hydrogen (left) and deuterium (right) burning mass limits.

extension of that of brown dwarfs. To determine the existence of the hypothetical opacity-limit cut-off we need both ultra-deep imaging with high sensitive facilities extending from 1 to $8 \mu \mathrm{m}$ as well as intermediate resolution spectroscopy, so that we can detect and confirm fainter isolated-planetary mass objects of a few Jupiter masses and assess their membership in the cluster.

\section{Summary}

We have performed a $790 \mathrm{arcmin}^{2}$-wide survey close to the centre of the $\sigma$ Orionis cluster $\left(3 \pm 2 \mathrm{Ma} ; 360_{-60}^{+70} \mathrm{pc}\right)$ in the $I$ - and $J$-bands down to limiting magnitudes $(3 \sigma) I \sim 24.1 \mathrm{mag}$ and $J \sim$ $21.8 \mathrm{mag}$. We selected 49 objects from the $I$ vs. $I-J$ diagram. Of these, 33 are brown dwarfs and 11 are planetary-mass objects at the most probable age and distance of the cluster. Twenty objects display spectroscopic features of youth. Two brown dwarfs and two planetary-mass object candidates are reported here for the first time.

The infrared follow-up from the $H$-band to $8.0 \mu \mathrm{m}$ with the 2MASS catalogue, deep ground-based near-infrared imaging with 2- and 4-m-class telescopes, and Spitzer Space Telescope archival infrared images did not allow us to detect any blue interlopers in our sample. However, we could use these data to identify 18 objects with flux excess at $8.0 \mu \mathrm{m}$ which are therefore likely to harbour discs. Some of them are T Tauri substellar analogs with discs. The frequency of brown dwarfs with discs in $\sigma$ Orionis is estimated at $47 \pm 9 \%$.

Taking into account the spectroscopic information and the IRAC photometry, 30 targets are confirmed as very young lowmass objects members of the cluster. Among the remaining 19 cluster candidates, up to six could be foreground contaminants (especially effecting the faintest magnitude interval in our sample).

We find a rising mass spectrum $\left(\Delta N / \Delta M \propto M^{-\alpha}\right)$ in the mass interval 0.11 and $0.006 M_{\odot}$. The $\alpha$ index is $+0.6 \pm 0.2$ for the whole mass interval considered, and $+0.4 \pm 0.2$ if we restrict it to the substellar domain between 0.073 and $0.006 M_{\odot}$. A break in the luminosity function is apparent at about $J \sim 16.0 \mathrm{mag}$ 
( $\sim 0.03 M_{\odot}$ at the most probable age of the cluster). Within the mass interval covered by our survey, there is no direct evidence for the presence of an opacity-mass limit for objects formed via fragmentation and collapse of molecular clouds. Any possible mass cut-off would lie below $6 M_{\text {Jup }}\left(0.006 M_{\odot}\right)$. Both brown dwarfs and IPMOs seem to form as an extension of the low-mass star formation process.

Acknowledgements. We thank the anonymous referee for his/her detailed and careful report, J. Hernández for helpful discussion on Spitzer data, and A Manchado, J. A. Acosta and the rest of the LIRIS instrument team for acquiring some deep $H$ and $K_{\mathrm{s}}$ data. Partial financial support was provided by the Spanish Ministerio de Ciencia y Tecnología proyect PNAYA 2006-12612 of the Plan Nacional de Astronomía y Astrofísica. Based on observations obtained at the Paranal Observatory, Chile, in ESO program 68.C-0553(A). Based on observations obtained at the Canada-France-Hawaii Telescope (CFHT) which is operated by the National Research Council of Canada, the Institut National des Sciences de l'Univers of the Centre National de la Recherche Scientifique of France, and the University of Hawai'i. Based on observations collected at the Centro Astronómico Hispano Alemán (CAHA) at Calar Alto, operated jointly by the Max-Planck Institut für Astronomie and the Instituto de Astrofísica de Andalucía (CSIC). Based on observations made with the Isaac Newton Telescope (INT) operated on the island of La Palma by the Isaac Newton Group in the Spanish Observatorio del Roque de Los Muchachos of the Instituto de Astrofísica de Canarias. The Telescopio Carlos Sánchez is operated on the island of Tenerife by the Instituto de Astrofísica de Canarias in the Spanish Observatorio del Teide of the Instituto de Astrofísica de Canarias. This work is based in part on observations made with the Spitzer Space Telescope, which is operated by the Jet Propulsion Laboratory, California Institute of Technology under a contract with NASA. This publication makes use of data products from the Two Micron All Sky Survey, which is a joint project of the University of Massachusetts and the Infrared Processing and Analysis Center/California Institute of Technology, funded by the National Aeronautics and Space Administration and the National Science Foundation. IRAF is distributed by National Optical Astronomy Observatories, which are operated by the Association of Universities for Research in Astronomy, Inc., under cooperative agreement with the National Science Foundation. This research has made use of the SIMBAD database, operated at CDS, Strasbourg, France.

\section{References}

Allard, F., Hauschildt, P. H., Alexander, D. R., Tamanai, A., \& Schweitzer, A. 2001, ApJ, 556, 357

Baraffe, I., Chabrier, G., Allard, F., \& Hauschildt, P. H. 1998, A\&A, 337, 403

Baraffe, I., Chabrier, G., Allard, F., \& Hauschildt, P. H. 2002, A\&A, 382, 563

Baraffe, I., Chabrier, G., Barman, T., Allard, F., \& Hauschildt, P. H. 2003, A\&A, 402,701

Barrado y Navascués, D., \& Martín, E. L. 2003, AJ, 126, 2997

Barrado y Navascués, D., Zapatero Osorio, M. R., Béjar, V. J. S., et al. 2001, A\&A, 377, L9

Barrado y Navascués D., Zapatero Osorio M. R., Martín E. L., et al. 2002, A\&A, 393, L85

Barrado y Navascués, D., Béjar, V. J. S., Mundt, R., et al. 2003, A\&A, 404, 171

Barrado y Navascués, D., Stauffer, J. R., Bouvier, J., Jayawardhana, R., \& Cuillandre, J.-C. 2004, ApJ, 610, 1064

Bate, M. R., Bonnell, I. A., \& Bromm, V. 2002, MNRAS, 332, L65

Bate, M. R., Bonnell, I. A., \& Bromm, V. 2003, MNRAS, 339, 577

Beaulieu, J.-P., Bennett, D. P., Fouqué, P., et al. 2006, Nature, 439, 437

Béjar, V. J. S., Zapatero Osorio, M. R., \& Rebolo, R. 1999, ApJ, 521, 671

Béjar, V. J. S., Martín, E. L., Zapatero Osorio, M. R., et al. 2001, ApJ, 556, 830

Béjar, V. J. S., Zapatero Osorio, M. R., \& Rebolo, R. 2004, AN, 325, 705

Bihain, G., Rebolo, R., Béjar, V. J. S., et al. 2006, A\&A, 458, 805

Boss, A. P. 2000, ApJ, 536, L101

Bouvier, J., Stauffer, J. R., Martín, E. L., et al. 1998, A\&A, 336, 490

Bouy, H., Duchêne, G., Köhler, R., et al. A\&A, 423, 341

Brown, A. G. A., de Geus, E. J., \& de Zeeuw, P. T. 1994, A\&A, 289, 101

Burningham, B., Naylor, T., Littlefair, S. P., \& Jeffries, R. D. 2005a, MNRAS, 356,1583

Burningham, B., Naylor, T., Littlefair, S. P., \& Jeffries, R. D. 2005b, MNRAS, 363,1389

Burrows, A., Marley, M., Hubbard, W. B., et al. 1997, ApJ, 491, 856

Caballero, J. A. 2005, Astron. Nachr., 326, 1007

Caballero, J. A. 2006, Ph.D. Thesis, Universidad de La Laguna

Caballero, J. A., Béjar, V. J. S., Rebolo, R., \& Zapatero Osorio, M. R. 2004, A\&A, 424, 857
Caballero, J. A., Martín, E. L., Zapatero Osorio, M. R., et al. 2006a, A\&A, 445, 143

Caballero, J. A., Martín, E. L., Dobbie, P. D., \& Barrado y Navascués, D. 2006b, A\&A, 460, 635

Caballero, J. A. 2007, A\&A, 466, 917

Chabrier, G., \& Baraffe, I. 2000, ARA\&A, 38, 337

Chabrier, G., Baraffe, I., Allard, F., \& Hauschildt, P. 2000, ApJ, 542, 464

Chauvin, G., Lagrange, A.-M., Dumas, C., et al. 2004, A\&A, 425, L29

Chauvin, G., Lagrange, A.-M., Dumas, C., et al. 2005, A\&A, 438, L25

Charbonneau, D., Brown, T. M., Latham, D. W., \& Mayor, M. 2000, ApJ, 529, L45

Chen, B., Stoughton, C., Smith, J. A., et al. 2001, ApJ, 553, 184

Cox A. N. 2000, Allen's astrophysical quantities, 4th ed. A. N. Cox (New York: AIP Press; Springer)

Cruz, K. L., Reid, I. N., Liebert, J., Kirkpatrick, J. D., \& Lowrance, P. J. 2003, AJ, 126, 2421

Cutri, R. M., Skrutskie, M. F., van Dyk, S., et al. 2003, VizieR On-line Data Catalog: II/246. Originally published in: University of Massachusetts and Infrared Processing and Analysis Center (IPAC/California Institute of Technology)

Cushing, M. C., Roellig, T. L., Marley, M. S., et al. 2006, ApJ, 648, 614

Dahn, C. C., Harris, H. C., Vrba, F. J., et al. 2002, AJ, 124, 1170

Dobbie, P. D., Pinfield, D. J., Jameson, R. F., \& Hodgkin, S. T. 2002, MNRAS, 335, L79

Douglas, J. N., Bash, F. N., Bozyan, F. A., Torrence, G. W., \& Wolfe, C. 1996, AJ, 111, 1945

Fernández, M., \& Comerón, F. 2001, A\&A, 380, 264

Flesch, E., \& Hardcastle, M. J. 2004, A\&A, 427, 387

Franciosini, E., Pallavicini, R., \& Sanz-Forcada, J. 2006, A\&A, 446, 501

Furlan, E., Calvet, N., D’Alessio, P., et al. 2005, ApJ, 621, L129

Golimowski, D. A., Leggett, S. K., Marley, M. S., et al. 2004, AJ, 127, 3516

Gonzalez-García, B. M., Zapatero Osorio, M. R., Béjar, V. et al. 2006, A\&A, 460,799

Greaves, J. S., Holland, W. S., \& Pound, M. W. 2003, MNRAS, 346, 441

Hernández, J., Hartmann, L., Megeath, T., et al. 2007, ApJ, accepted, [arXiv:astro-ph/0701476]

Hillenbrand, L. A., \& Hartmann, L. W. 1998, ApJ, 492, 540

Jameson, R. F. 2005, AN, 326, 874

Jayawardhana, R., Mohanty, S., \& Basri, G. 2003, ApJ, 592, 282

Jeffries, R. D., Maxted, P. F. L., Oliveira, J. M., \& Naylor, T. 2006, MNRAS, 317, L6

Karaali, S. 2006, AN, 327, 97

Kenyon, M. J., Jeffries, R. D., Naylor, T., Oliveira, J. M. \& Maxted, P. F. L. 2005, MNRAS, 356, 89

Kirkpatrick, J. D., McGraw, J. T., Hess, T. R., Liebert, J., \& McCarthy, D. W. Jr. 1994, ApJS, 94, 749

Köhler, R., Petr-Gotzens, M. G., McCaughrean, M. J., et al. 2006, A\&A, 458, 461

Kroupa, P. 2001, MNRAS, 322, 231

Landolt, A. 1992, AJ, 104, 340

Lee, T. A. 1968, ApJ, 152, 913

Lee, C. W., Martín, E. L., \& Mathieu, R. D. 1994, AJ, 108, 1445

Lucas, P. W., \& Roche, P. F. 2000, MNRAS, 314, 858

Lucas, P. W., Roche, P. F., \& Tamura, M. 2005, MNRAS, 361, 211

Luhman, K. L. 2000, ApJ, 544, 1044

Luhman, K. L., Whitney, B. A., Meade, M. R., et al. 2006, ApJ, 647, 1180

Martín, E. L., Zapatero Osorio, M. R., Barrado y Navascués, D., Béjar, V. J. S., \& Rebolo, R. 2001, ApJ, 558, L117

Martín, E. L., \& Zapatero Osorio, M. R. 2003, ApJ, 593, L113

McGovern, M. R., Kirkpatrick, J. D., McLean, I. S., et al. 2004, ApJ, 600, 1020

Mokler, F., \& Stelzer, B. 2002, A\&A, 391, 1025

Mayor, M., \& Queloz, D. 1995, Nature, 378, 355

Muench, A. A., Lada, E. A., Lada, C. J., \& Alves, J. 2002, ApJ, 573, 366

Muench, A. A., Lada, E. A., Lada, C. J., et al. 2003, AJ, 125, 2029

Muzerolle, J., Hillenbrand, L., Calvet, N., Briceño, C., \& Hartmann, L. 2003, ApJ, 592, 266

Najita, J. R., Tiede, G. P., \& Carr, J. S. 2000, ApJ, 541, 977

Nakajima, T. 2005, IAC/TNG Workshop on Ultralow-mass star formation and evolution, La Palma, 2005 June 28 - July 1, ed. E. L. Martín \& A. Magazzù

Natta, A., \& Testi, L. 2001, A\&A, 376, L22

Neuhäuser, R., Guenther, E. W., Wuchterl, G., et al. 2005, A\&A, 435, L13

Oasa, Y., Tamura, M., Nakajima, Y., et al. 2006, AJ, 131, 1608

Oliveira, J. M., Jeffries, R. D., Kenyon, M. J., Thompson, S. A., \& Naylor, T. 2002, A\&A, 382, L22

Oliveira, J. M., Jeffries, R. D., van Loon, J. Th., \& Rushton, M. T. 2006, MNRAS, 369, 272

Patten, B. M., Stauffer, J. R., Burrows, A., et al. 2006, ApJ, 651, 502

Perryman, M. A. C., Brown, A. G. A., Lebreton, Y., et al. 1998, A\&A, 331, 81 
Phleps, S., Drepper, S., Meisenheimer, K. \& Fuchs, B. 2005, A\&A, 443, 929 Pinfield, D. J., Jameson, R. F., \& Hodgkin, S. T. 1998, MNRAS, 299, 955 Pinfield, D. J., Dobbie, P. D., Jameson, R. F., et al. 2003, MNRAS, 342, 1241 Rees, M. J. 1976, MNRAS, 176, 483

Reipurth, Bo \& Clarke, C. 2001, AJ, 122, 432

Ryan, Jr. R. E., Hathi, N. P., Cohen, S. H., \& Windhorst, R. A. 2005, ApJ, 631, L159

Scalo, J. M. 1986, Fund. Cos. Phys., 11, 1

Scholz, A., \& Eislöffel, J. 2004, A\&A, 419, 249

Sherry, W. H., Walter, F. M., \& Wolk, S. J. 2004, AJ, 128, 2316

Silk, J. 1977, ApJ, 214, 152

Slesnick, C. L., Hillenbrand, L. A., \& Carpenter, J. M. 2004, ApJ, 610, 1045

Stassun, K. G., Mathieu, R. D., \& Valenti, J. A. 2006, Nature, 440, 311

Tohline, J. E. 1980, ApJ, 239, 417

Tsuji, T., Ohnaka, K., Aoki, W., \& Nakajima, T. 1996, A\&A, 308, L29

Vrba, F. J., Henden, A. A., Luginbuhl, C. B., et al. 2004, AJ, 127, 2948
White, R. J., \& Basri, G. 2003, ApJ, 582, 1109

Wilking, B. A., Greene, T. P., \& Meyer, M. R. 1999, AJ, 117, 469

Zapatero Osorio, M. R., Rebolo, R., \& Martín, E. L. 1997, A\&A, 317, 164

Zapatero Osorio, M. R., Béjar, V. J. S., Rebolo, R., Martín, E. L., \& Basri, G. 1999, ApJ, 524, L115

Zapatero Osorio, M. R., Béjar, V. J. S., Martín, E. L., et al. 2000, Science, 290, 103

Zapatero Osorio, M. R., Béjar, V. J. S., Pavlenko, Ya., et al. 2002a, A\&A, 384, 937

Zapatero Osorio, M. R., Béjar, V. J. S., Martín, E. L., Barrado y Navascués, D., \& Rebolo, R. 2002b, ApJ, 569, L99

Zapatero Osorio, M. R., Béjar, V. J. S., Martín, E. L., et al. 2002c, ApJ, 578, 536

Zapatero Osorio, M. R., Caballero, J. A., Béjar, V. J. S., \& Rebolo, R. 2003, A\&A, 408, 663

Zapatero Osorio, M. R., Lane, B. F., Pavlenko, Ya., et al. 2004, ApJ, 615, 958 
J. A. Caballero et al.: The substellar mass function in $\sigma$ Orionis. II., Online Material $p 1$

\section{Online Material}




\section{Appendix A: Other interesting sources}

\section{A.1. SOri J053948.1-022914}

S Ori J053948.1-022914 was discovered by Béjar et al. (2001). It is a M7.0 \pm 0.5 object with a stable chromospheric $\mathrm{H} \alpha$ emission $(\mathrm{p} E W(\mathrm{H} \alpha)=-6.7 \pm 1.6 \AA$; Barrado y Navascués et al. 2003) and with a very red $J-K_{\mathrm{s}}$ colour for its spectral type $\left(J-K_{\mathrm{s}}=1.28 \pm 0.17 \mathrm{mag}\right)$. It was proposed to be a X-ray source by Mokler \& Stelzer (2002) from ROSAT data. Flesch \& Hardcastle (2004) correlated, however, the X-ray source with a brighter star at 10.2 arcsec detected to the northeast in their APM/USNO-A search. This star, B 1.01-319, displays low gravity features and radial velocity consistent with membership in $\sigma$ Orionis (Burningham et al. 2005a). Therefore, the X-ray emission is likely ascribed to the star, at a projected physical separation of about $3700 \mathrm{AU}$ to the M7.0 object.

Scholz \& Eislöffel (2004) detected a non-periodic photometric variability with a $r m s$ of $0.139 \mathrm{mag}$ in the red-optical light curve of S Ori J053948.1-022914. The average I magnitude measured by them, $18.10 \mathrm{mag}$, considerably differs from other measurements in the literature $(I=18.92 \mathrm{mag}$ in Béjar et al. $1999 ; I=18.67 \pm 0.07 \mathrm{mag}$ in Béjar et al. $2004 ; I=18.50 \mathrm{mag}$ in this work). It might be a photometrically-variable cluster member. Further studies are needed to assess its membership in $\sigma$ Orionis.

\section{A.2. New probable cluster non-members}

In Table A. 1 we provide the coordinates and $I J H K_{\mathrm{s}}$ magnitudes of four interesting red objects that probably do not belong to the $\sigma$ Orionis cluster. They are bluewards of our selection criterion. Two of them, with identifications 1 and 2 in the table, are likely L-type field ultracool dwarfs in the foreground. Their $J-K_{\mathrm{s}}$ and $I-J$ colours match the sequence of the ultracool field dwarfs depicted in the colour-colour diagram in the bottom right panel in Fig. 4. The sources with identifications 3 and 4 are likely of extragalactic nature. Interestingly, the object Id. 3 is located at only $1.5 \pm 1.0$ arcsec to the radio source TXS 0537-029 (Douglas et al. 1996). Given the low spatial density of radio sources towards $\sigma$ Orionis, it is possible that Id. 3 and the radio source are associated with a non-catalogued elliptical galaxy 6.2 arcsec to the southeast of Id. 3 . 
J. A. Caballero et al.: The substellar mass function in $\sigma$ Orionis. II., Online Material $p 3$

Table A.1. New probable cluster non-members.

\begin{tabular}{cccccccc}
\hline \hline Id. & $\alpha^{J 2000}$ & $\delta^{J 2000}$ & $I \pm \delta I(\mathrm{mag})$ & $J \pm \delta J(\mathrm{mag})$ & $H \pm \delta H(\mathrm{mag})$ & $K_{\mathrm{s}} \pm \delta K_{\mathrm{s}}(\mathrm{mag})$ & Remarks \\
\hline 1 & 053925.4 & -022943 & $23.11 \pm 0.18$ & $20.08 \pm 0.10$ & $19.43 \pm 0.07$ & $19.07 \pm 0.07$ & Early L-type field dwarf? \\
2 & 053844.6 & -024440 & $23.8 \pm 0.5$ & $20.64 \pm 0.08$ & $19.90 \pm 0.09$ & $19.22 \pm 0.10$ & Late L-type field dwarf? \\
3 & 053959.3 & -025256 & $23.3 \pm 0.3$ & $20.82 \pm 0.09$ & $19.6 \pm 0.2$ & $18.65 \pm 0.07$ & Radio galaxy? \\
4 & 054004.7 & -022927 & $\approx 24.5$ & $21.56 \pm 0.10$ & $20.01 \pm 0.12$ & $18.52 \pm 0.13$ & Extremely red galaxy? \\
\hline
\end{tabular}




\section{Appendix B:}

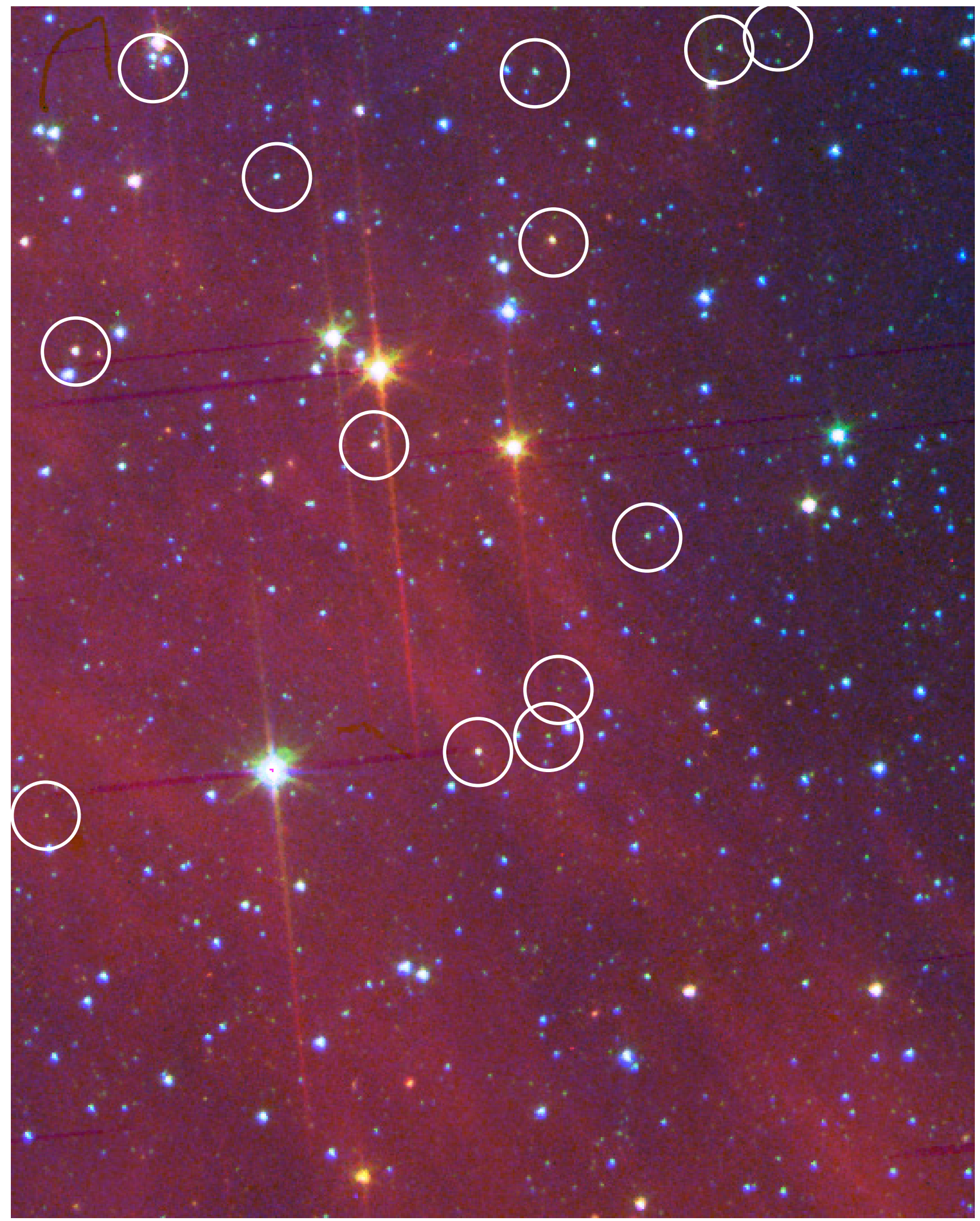

Fig. B.1. False-colour composite image of the northern part of survey area corresponding to the detector CCD\#2 of the WFC ( 11 arcmin wide; north is up, east is left). Blue is for photographic $I$ (from the Canadian Astronomy Data Centre), green is for IRAC $3.6 \mu \mathrm{m}$ and red is for IRAC 5.8 $\mu \mathrm{m}$. The two very low-mass stars and the eleven brown dwarfs in the area are marked with white circles. The brightest blueish star is HD 294278, a K2-type foreground dwarf. The remaining bright greenish and orange stars are known classical T Tauri stars in $\sigma$ Orionis, like TX Ori and V505 Ori. 